Check for updates

Cite this: RSC Adv., 2019, 9, 33170

Received 11th September 2019

Accepted 9th October 2019

DOI: $10.1039 / c 9 r a 07288 h$

rsc.li/rsc-advances

\section{Heterocyclic iodoniums as versatile synthons to approach diversified polycyclic heteroarenes $\uparrow$}

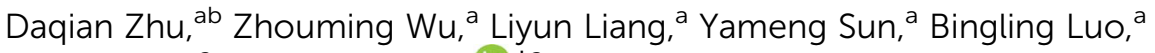 \\ Peng Huang ${ }^{a}$ and Shijun Wen (D) *a
}

\begin{abstract}
Polycyclic heteroarenes are important scaffolds in the construction of pharmaceuticals. We have previously developed a series of novel heterocyclic iodoniums. In our current work, these unique iodoniums were employed to construct various complex polycyclic heteroarenes with structural diversity via tandem dual arylations. As a result, indole, thiophene and triphenylene motifs were fused into these heterocycles with high molecular quality, which might provide promising fragments in drug discovery. Moreover, these heterocycles could be diversified at a late stage.
\end{abstract}

\section{Introduction}

Polycyclic heteroarenes are important scaffolds in the construction of pharmaceuticals. ${ }^{1}$ Compared with polycyclic aromatic hydrocarbons, heterocycles exhibit improved solubility and bioavailability, which make them promising drug candidates. ${ }^{2}$ Many heterocycles are reported as kinase inhibitors, anti-infective and antibacterial agents (Fig. 1). Thus, the design and synthesis of polycyclic heteroarenes are highly demanded.

Tandem reactions enable rapid access to complex molecules, avoiding tedious purification steps and minimizing chemical waste generation. ${ }^{3}$ As a paradigm of tandem reactions, dual arylations with cyclic diphenyl iodoniums (CDPIs) are employed to construct various polycycles, such as acridine ${ }^{4}$ carbazole,${ }^{5}$ fluorene, ${ }^{6}$ phenanthrene, ${ }^{7}$ and dibenzothiophene. ${ }^{8}$ These obtained polycycles are often heavily hydrocarbon oriented. In the drug discovery field, heterocyclic frameworks are crucial to gain druggability. Heterocyclic iodoniums (HCIs) could be promising alternative reagents to replace CDPIs for the potential construction of heterocycles (Scheme 1). However, HCIs are under-explored and only few of them have been reported. ${ }^{9}$ Very recently, we have developed a series of new HCIs, ${ }^{10}$ and now we wish to fully investigate their synthetic application potentials to obtain diverse heterocycles via tandem transformations.

Indole-fused polyheterocycles are privileged structural motifs. ${ }^{11}$ Despite various strategies to generate these complex

${ }^{a}$ State Key Laboratory of Oncology in South China, Collaborative Innovation Center for Cancer Medicine, Sun Yat-sen University Cancer Center, 651 Dongfeng East Road, Guangzhou 510060, China.E-mail: wenshj@sysucc.org.cn

${ }^{b}$ School of Pharmacy, Guangdong Pharmaceutical University, 280 Waihuan East Road, Guangzhou 510006, China

$\dagger$ Electronic supplementary information (ESI) available. See DOI: 10.1039/c9ra07288h molecules, there still lacks a general approach to rapidly build libraries of indole-fused heterocycles with a skeleton diversity. In our previous work, dual aminations of CDPIs led to the construction of functionalized $\mathrm{N}$-substituted carbazoles. ${ }^{5 b}$ Inspired by this work, we hypothesized the amination strategy could be extended to construct indole-fused heterocycles if HCIs were used as starting materials to replace CDPIs. In this current work, we thoroughly investigated tandem dual aminations of HCIs with various amines to produce indole-fused polycyclic scaffolds. In addition, the annulations of HCIs with triethylammonium $N$-benzyldithiocarbamate and 2-chlorobenzoic acid were also fully investigated. These transformations will provide efficient pathways for rapid generation of complex heterocycles with a structural diversity.

\section{Results and discussion}

Chromone is a privileged motif for drug design and discovery in the field of medicinal chemistry. ${ }^{12}$ Thus, we commenced the dual-amination transformation using chromone embedded HCIs 1a-1e as building blocks (Scheme 2). Under catalytic mediation of $\mathrm{Cu}(\mathrm{OAc})_{2}, p$-anisidine underwent dual arylations to complete the amination.

As a result, the desired chromone-fused indoles were obtained at modest to good yields (3a-3e). Then, we explored the substrate scope and generality of other HCIs with different heterocyclic motifs. Thiochromone-fused indole was obtained at a moderate yield (3f). Quinoline, isoquinoline and coumarin are important building blocks for naturally occurring products and pharmaceuticals. These unique heterocyclic fused indoles could be also assembled efficiently $(\mathbf{3 g}-\mathbf{3 m})$. The HCIs bearing chlorine atom usually gave low yields (3a vs. $3 \mathbf{c}, 3 \mathbf{g} v s .3 \mathbf{i})$. Meanwhile, the construction of thieno[3,2-b]indole (3n) was realized, providing a concise method to obtain thiophenecontaining materials. 
<smiles>O=C(O)Cc1cccc2c(=O)c3[nH]c4ccccc4oc=3c12</smiles>

Benzopyranoindole Aminopeptidase N/CD13 inhibitor Chk1 inhibitors<smiles>O=c1oc2cc(O)ccc2c2c1[nH]c1ccc(O)cc12</smiles>

Chromenoindole Topoisomerase I inhibitor
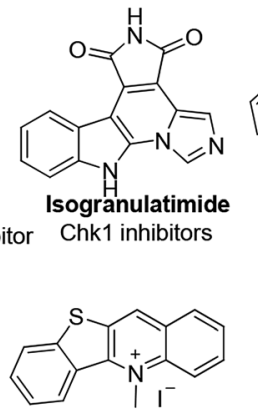

Benzothienoquinoline Anti-infective agent
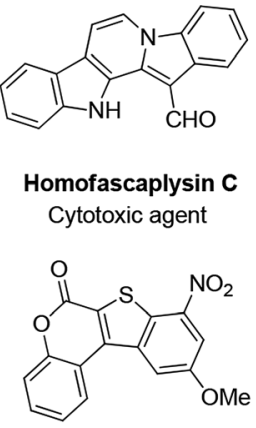

Benzothiocoumarin Antibacterial agent
Fig. 1 Selected examples of heterocyclic natural products and pharmaceuticals.

Subsequently, the substrate scope of this reaction was further examined on variation of the amines (Scheme 3). Like $p$ anisidine, other arylamines bearing different functional groups also enabled their incorporation into chromone-containing HCI 1a to provide diverse chromone-fused indoles (4a-4i). It should be noted that the intact bromo or iodo in products $4 \mathbf{e}$ and $\mathbf{4 f}$ could serve as a potential transformation platform for late-stage diversification. Meanwhile, arylamines with electron-deficient groups disfavored these reactions and provided the products in low yields $(\mathbf{4 h}-\mathbf{4 j})$. Amines tethering on pyridine and quinoline also performed smoothly $(\mathbf{4 j} \mathbf{j} \mathbf{4 k})$. However, additional $\mathrm{CuI}$ (0.1 equiv.) was required while alkyl amines were used. Under the modified condition, the desirable products were successfully obtained (41-4o). Again, pyridine motif in the alkylamine did not disrupt the reaction (41).

Sulfur containing heterocycles have found considerable utility particularly in material science because of high resonance energy of sulfur atom. ${ }^{\mathbf{1 3}}$ Traditional methods for the introduction of sulfur suffer from several disadvantages such as catalyst poisoning, over-oxidization, and stinky smell. Using our recently discovered odor-free triethylammonium $\mathrm{N}$-benzyldithiocarbamate (M1) as the sulfur source donor, ${ }^{8 a}$ reactions of HCIs and M1 under mediation of copper sulfate could smoothly furnish benzothiophene-fused heterocyclic frameworks, including chromone (5a), quinoline (5b), isoquinoline (5c) and coumarin (5d), as shown in Scheme 4.

Decarboxylation of commercially available carboxylic acids is emerging as a novel strategy for aromatic functionalization. ${ }^{\mathbf{1 4}} \mathrm{A}$ pioneering work has recently been extended to decarboxylation of 2-chlorobenzoic acid for in situ generation of benzyne to

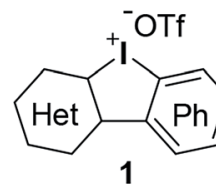

Heterocyclic iodoniums (HCls)
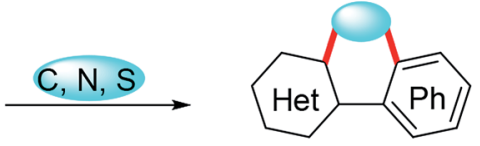

Polycyclic heteroarenes construct triphenylenes. ${ }^{15}$ As counterparts of these hydrocarbons, the heteroatom-containing triphenylene analogues exhibit distinct chemical and physical properties. ${ }^{\mathbf{1 6}}$ However, they have been so far less touched due to limited synthetic protocols. Thus, HCIs could be very promising synthons to construct such unique triphenylene. In our study, HCIs reacted with 2-chlorobenzoic acid to effectively afford the desirable annulated heterocycles containing chromone (6a), thiochromone (6b), or quinoline (6c), as shown in Scheme 5.

Finally, we have taken several applications to further demonstrate the robustness of these unique heterocyclic iodoniums as synthons (Scheme 6). Firstly, the copper-catalyzed

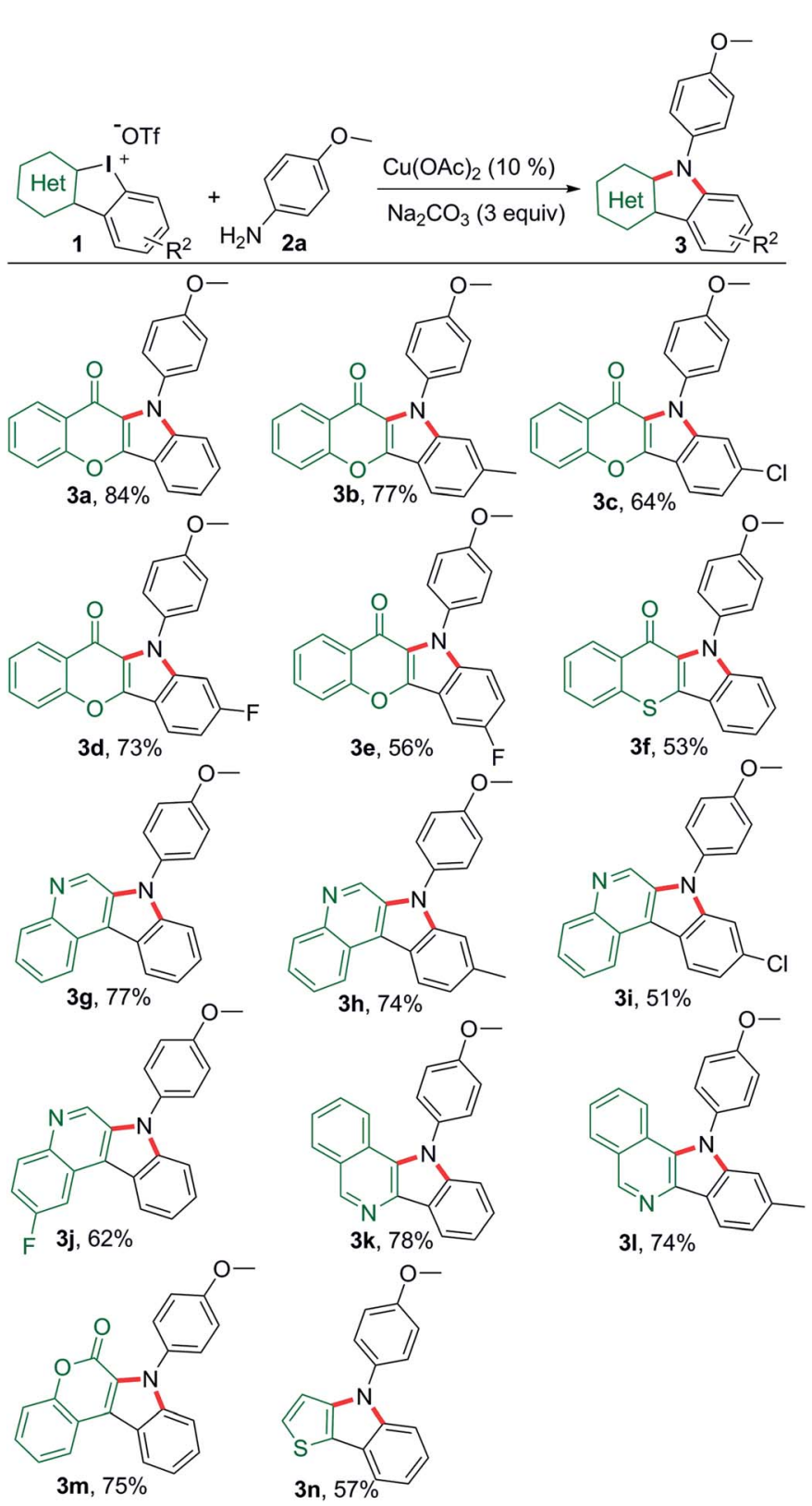

Scheme 2 Substrate scope of $\mathrm{HCls}$ to synthesize heterocycle-fused indoles. Reaction conditions: 1 ( $0.1 \mathrm{mmol}), p$-anisidine (2.5 equiv.), $i-$ $\mathrm{PrOH} /\left(\mathrm{CH}_{2} \mathrm{OH}\right)_{2}(0.9 / 0.1 \mathrm{~mL})$, refluxing, $\mathrm{Ar}, 16 \mathrm{~h}$.

Scheme 1 Synthesis of polycyclic heteroarenes using $\mathrm{HCls}$. 


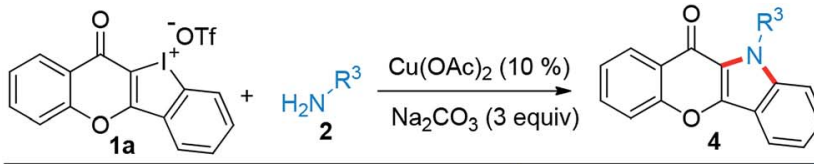<smiles>O=c1c2ccccc2oc2c1c1ccccc1n2-c1ccccc1</smiles><smiles>Cc1ccc(-n2c3ccccc3c3oc4ccccc4c(=O)c32)cc1</smiles>

4a, $79 \%$<smiles></smiles>

4b, $92 \%$

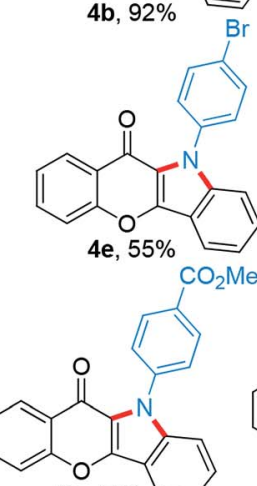

4h, $41 \%$<smiles>O=c1c2ccccc2oc2c3ccccc3n(-c3ccccn3)c12</smiles>
4j, $34 \%$<smiles>CCCCn1c2ccccc2c2oc3ccccc3c(=O)c21</smiles>

$4 \mathrm{~m}, 68 \%^{\mathrm{a}}$<smiles>O=c1c2ccccc2oc2c1c1ccccc1n2-c1cccc2cccnc12</smiles>

4k, $67 \%$<smiles>O=c1c2ccccc2oc2c3ccccc3n(C3CC3)c12</smiles>

4n, $82 \%$<smiles></smiles><smiles></smiles><smiles>C/C=C\[14CH2][14CH3]</smiles><smiles>C=CC(I)=CC</smiles><smiles></smiles><smiles>CCCn1c2ccccc2c2oc3ccccc3c(=O)c21</smiles>

$41,68 \%$<smiles>O=c1c2ccccc2oc2c3ccccc3n(CCCCO)c12</smiles>

Scheme 3 Scope of amines reacting with 1a to construct chromonefused indoles. Reaction conditions: 1 a $(0.1 \mathrm{mmol})$, amine (2.5 equiv.), i$\mathrm{PrOH} /\left(\mathrm{CH}_{2} \mathrm{OH}\right)_{2}(0.9 / 0.1 \mathrm{~mL})$, refluxing, $\mathrm{Ar}, 16 \mathrm{~h}$. ${ }^{\mathrm{a}}$ Additional $\mathrm{Cul}(0.1$ equiv.) added.

dual aminations of HCIs performed well in a gram-scale reaction without a compromised yield (Scheme 6a). The increasing emergence of drug resistance in treating diseases demands an urgent need to develop new drugs. One effective strategy has been pursued combining two different drugs to form a new

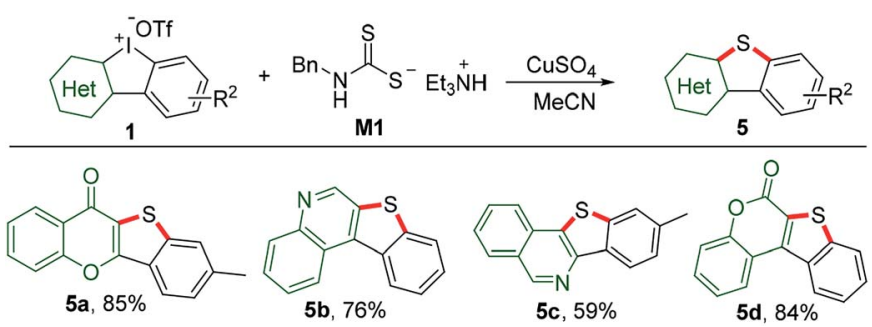

Scheme 4 Sulfur insertion of $\mathrm{HCls}$ with triethylammonium $\mathrm{N}$-benzyldithiocarbamate M1. Reaction conditions: 1 (1 equiv.), M1 (2 equiv.), $\mathrm{CuSO}_{4}$ (0.1 equiv.), $\mathrm{MeCN}, 60{ }^{\circ} \mathrm{C}, \mathrm{Ar}, 6 \mathrm{~h}$.

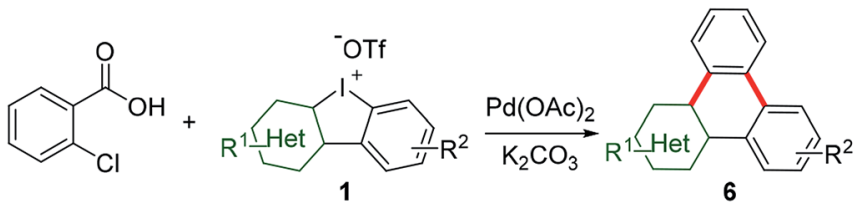<smiles>Cc1ccc2c(c1)c1ccccc1c1c(=O)c3ccccc3oc21</smiles><smiles></smiles><smiles>Fc1ccc2ncc3c4ccccc4c4ccccc4c3c2c1</smiles>

Scheme 5 Annulation of 2-chlorobenzoic acid with $\mathrm{HCls}$. Reaction conditions: 2-chorobenzoic acid (1 equiv.), 1 (1.2 equiv.), $\mathrm{K}_{2} \mathrm{CO}_{3}$ (2.2 equiv.), $\mathrm{Pd}(\mathrm{OAc})_{2}$ (0.1 equiv.), 1-methyl-2-pyrrolidinone, $140{ }^{\circ} \mathrm{C}, 16 \mathrm{~h}$.

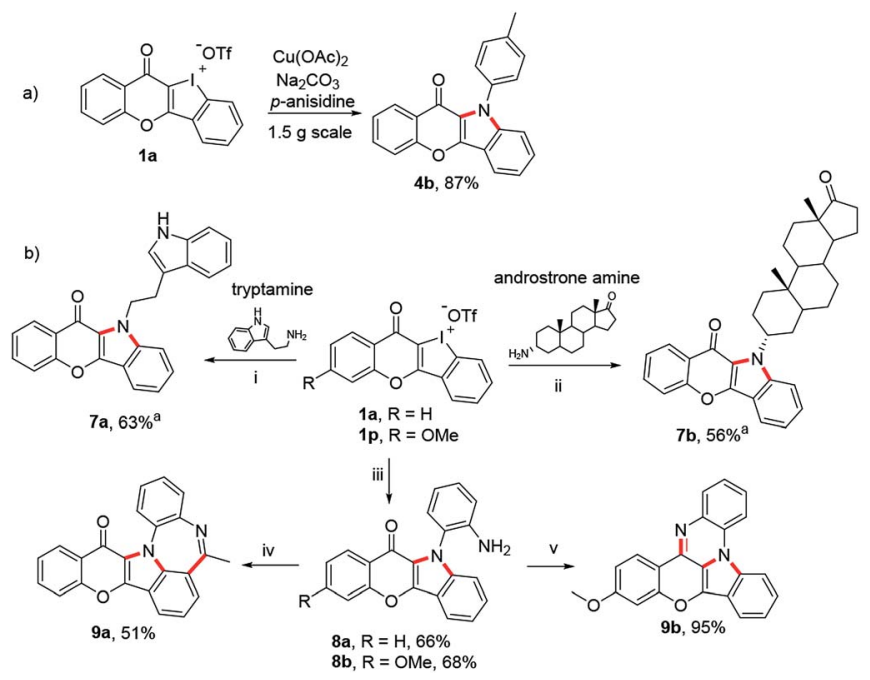

Scheme 6 (a) Scale-up synthesis of $4 \mathrm{~b}$ under the standard condition. (b) Synthesis of drug-like hybrids and late-stage diversification with $1 \mathrm{a}$ and 1p. Reaction conditions: (i)-(iii) 1a or 1p (0.2 mmol), amine (2.5 equiv.), $\mathrm{Na}_{2} \mathrm{CO}_{3}$ (3 equiv.), $\mathrm{Cu}(\mathrm{OAc})_{2}$ (0.1 equiv.), $i-\mathrm{PrOH} /\left(\mathrm{CH}_{2} \mathrm{OH}\right)_{2}$ (1.8/0.2 mL), Ar, refluxing, $16 \mathrm{~h}$. (iv) $\mathrm{AcCl}$ (1.2 equiv.), $\mathrm{Et}_{3} \mathrm{~N}$ (2.0 equiv.), $\mathrm{CH}_{2} \mathrm{Cl}_{2}$, rt; PPA $(0.2 \mathrm{~mL}), \mathrm{POCl}_{3}$ (10 equiv.), $120^{\circ} \mathrm{C}$. (v) $\mathrm{TsOH}-\mathrm{H}_{2} \mathrm{O}(0.1$ equiv.), EtOH, reflux. ${ }^{a}$ Additional $\mathrm{Cul}$ (0.1 equiv.) added.

hybrid molecule. ${ }^{\mathbf{1 7}}$ Herein, tryptamine, a serotonin receptor agonist, and an amine derived from androstrone (an endogenous steroid hormone) were employed to react with chromonefused HCI 1a under the standard conditions. Both transformations successfully provided the expected fused hybrids $7 \mathbf{a}$ and $\mathbf{7 b}$. In a final venture to establish the generality of this strategy, benzene-1,2-diamine was also used to prepare 8a and $\mathbf{8 b}$ which were readily for further transformation to obtain more complex heteropolycycles $\mathbf{9 a}$ and $\mathbf{9 b}$ with potential druglikeness (Scheme 6b).

\section{Conclusions}

In conclusion, we have fully explored the synthetic application of our recently reported heterocyclic iodoniums (HCIs). These unique iodoniums may gain more attention to build complex 
polycyclic heteroarenes which are widely present in naturally occurring products and pharmaceuticals. All the transformations with HCIs underwent a cyclization to build structurally diverse indole-fused, thiophene-fused, and triphenylenefused heterocycles. Our current thorough investigation of HCIs highlights their value as versatile building blocks in synthetic chemistry, which may provide novel structures for drug development. These particular heterocycles are currently under our anticancer drug screening.

\section{Experimental}

The ${ }^{1} \mathrm{H}$ and ${ }^{13} \mathrm{C}$ nuclear magnetic resonance (NMR) spectra were recorded on a Bruker Avance spectrometer 400 at $400 \mathrm{MHz}$ and $100 \mathrm{MHz}$, respectively. Chemical shifts are given in ppm $(\delta)$ referenced to $\mathrm{CDCl}_{3}$ with 7.26 for ${ }^{1} \mathrm{H}$ and 77.10 for ${ }^{13} \mathrm{C}$, and to $d_{6}$-DMSO with 2.50 for ${ }^{1} \mathrm{H}$ and 39.5 for ${ }^{13} \mathrm{C}$. In the case of multiplet, signals are reported as intervals and abbreviated as follows: s, singlet; d, doublet; t, triplet; q, quartet; m, multiplet. Coupling constants are expressed in hertz. High-resolution mass spectra (HRMS) were recorded on a BRUKER VPEXII spectrometer with ESI mode unless otherwise stated. Melting point was measured by BUCHI Melting Point B-540. The progress of the reactions was monitored by thin-layer chromatography on a glass plate coated with silica gel with fluorescent indicator (GF254). Column chromatography was performed on silica gel (200-300 mesh).

\section{General procedure $A$ for the synthesis of $3 a-4 k$}

The synthesis of 10-(4-methoxyphenyl)chromeno[3,2- $b]$ indol$11(10 H)$-one (3a) is exemplified. To a stirred solution of iodonium $1 \mathrm{a}(0.1 \mathrm{mmol})$ in $i$-PrOH $(0.9 \mathrm{~mL})$, was added ethylene glycol ( $0.1 \mathrm{~mL}$ ), $p$-anisidine (2.5 equiv.), $\mathrm{Na}_{2} \mathrm{CO}_{3}$ (3 equiv.), and $\mathrm{Cu}(\mathrm{OAc})_{2}(0.1$ equiv.). The reaction proceeded at reflux for $16 \mathrm{~h}$ under argon atmosphere before $i$-PrOH was removed by a rotary evaporation. The remained mixture was extracted with EtOAc. The combined organic layers were washed with $\mathrm{H}_{2} \mathrm{O}$ and brine, dried over anhydrous $\mathrm{Na}_{2} \mathrm{SO}_{4}$, and evaporated in a vacuo. The residue was purified by column chromatography $(\mathrm{PE} / \mathrm{EtOAc}=$ $15 / 1-5 / 1$ ) to provide $3 \mathrm{a}$ as a white solid ( $29 \mathrm{mg}, 84 \%$ yield), $\mathrm{mp}$ 199.1-200.6 ${ }^{\circ} \mathrm{C} .{ }^{1} \mathrm{H}$ NMR $\left(400 \mathrm{MHz}, \mathrm{CDCl}_{3}\right) \delta 8.37(\mathrm{~d}, J=8.0 \mathrm{~Hz}$, $1 \mathrm{H}), 8.09(\mathrm{~d}, J=8.0 \mathrm{~Hz}, 1 \mathrm{H}), 7.74-7.68(\mathrm{~m}, 2 \mathrm{H}), 7.51-7.45(\mathrm{~m}$, $1 \mathrm{H}), 7.44-7.37(\mathrm{~m}, 3 \mathrm{H}), 7.32(\mathrm{dd}, J=13.2,7.9 \mathrm{~Hz}, 2 \mathrm{H}), 7.11-7.04$ (m, 2H), $3.91(\mathrm{~s}, 3 \mathrm{H}) \mathrm{ppm} .{ }^{13} \mathrm{C}$ NMR $\left(100 \mathrm{MHz}, \mathrm{CDCl}_{3}\right) \delta 169.2$, 159.3, 155.5, 146.0, 139.4, 132.8, 130.0, 129.1, 128.6, 126.4, 124.6, 124.0, 121.1, 120.9, 120.0, 118.1, 115.6, 114.3, 111.6, $55.6 \mathrm{ppm}$. HRMS (ESI) $\mathrm{m} / \mathrm{z}$ calcd for $\mathrm{C}_{22} \mathrm{H}_{16} \mathrm{NO}_{3}[\mathrm{M}+\mathrm{H}]^{+}$: 342.1125, found: 342.1112 .

10-(4-Methoxyphenyl)-8-methylchromeno[3,2-b]indol-11(10H)one (3b). $3 \mathbf{b}(27 \mathrm{mg}, 77 \%$ yield) was generated following a procedure for the synthesis of 3a as a white solid, mp 195.6-196.3 ${ }^{\circ} \mathrm{C} .{ }^{1} \mathrm{H}$ NMR $\left(400 \mathrm{MHz}, \mathrm{CDCl}_{3}\right) \delta 8.36(\mathrm{~d}, J=8.2 \mathrm{~Hz}, 1 \mathrm{H}), 7.95(\mathrm{~d}, J=$ $8.2 \mathrm{~Hz}, 1 \mathrm{H}), 7.69(\mathrm{~d}, J=2.2 \mathrm{~Hz}, 2 \mathrm{H}), 7.40(\mathrm{dd}, J=10.9,5.9 \mathrm{~Hz}, 3 \mathrm{H})$, $7.14(\mathrm{~d}, J=8.3 \mathrm{~Hz}, 1 \mathrm{H}), 7.11-7.04(\mathrm{~m}, 3 \mathrm{H}), 3.91(\mathrm{~s}, 3 \mathrm{H}), 2.48$ (s, 3H) ppm. ${ }^{13} \mathrm{C}$ NMR $\left(100 \mathrm{MHz}, \mathrm{CDCl}_{3}\right) \delta 168.9,159.3,155.5,146.3$, 140.0, 139.5, 132.6, 130.2, 129.2, 126.4, 124.7, 123.9, 123.2, 120.7,
119.7, 118.0, 114.3, 113.5, 111.1, 55.7, 22.5 ppm. HRMS (ESI) $\mathrm{m} / \mathrm{z}$ calcd for $\mathrm{C}_{23} \mathrm{H}_{18} \mathrm{NO}_{3}[\mathrm{M}+\mathrm{H}]^{+}: 356.1281$, found: 356.1284 .

8-Chloro-10-(4-methoxyphenyl)chromeno[3,2-b] indol-11(10H)one (3c). $3 \mathbf{c}(24 \mathrm{mg}, 74 \%$ yield) was generated following a procedure for the synthesis of $3 \mathrm{a}$ as a white solid, $\mathrm{mp} 215.1-216.4^{\circ} \mathrm{C} .{ }^{1} \mathrm{H}$ NMR $\left(400 \mathrm{MHz}, \mathrm{CDCl}_{3}\right) \delta 8.34(\mathrm{~d}, J=7.8 \mathrm{~Hz}, 1 \mathrm{H}), 7.99(\mathrm{~d}, J=$ $8.5 \mathrm{~Hz}, 1 \mathrm{H}), 7.75-7.64(\mathrm{~m}, 2 \mathrm{H}), 7.41(\mathrm{dd}, J=11.2,8.1 \mathrm{~Hz}, 3 \mathrm{H}), 7.30$ (s, 1H), $7.27(\mathrm{~d}, J=7.0 \mathrm{~Hz}, 1 \mathrm{H}), 7.08(\mathrm{~d}, J=8.7 \mathrm{~Hz}, 2 \mathrm{H}), 3.91$ (s, 3H) ppm. ${ }^{13} \mathrm{C}$ NMR (100 MHz, $\left.\mathrm{CDCl}_{3}\right) \delta$ 169.1, 159.6, 155.6, 145.6, 139.6, 134.8, 133.1, 129.5, 129.0, 126.4, 124.5, 124.3, 122.2, 121.4, 121.1, 118.1, 114.5, 114.2, 111.6, $55.7 \mathrm{ppm}$. HRMS (ESI) $\mathrm{m} / \mathrm{z}$ calcd for $\mathrm{C}_{22} \mathrm{H}_{15} \mathrm{ClNO}_{3}[\mathrm{M}+\mathrm{H}]^{+}: 376.0735$, found: 376.0742 .

8-Fluoro-10-(4-methoxyphenyl)chromeno[3,2-b]indol-11(10H)one (3d). 3d ( $26 \mathrm{mg}, 73 \%$ yield) was generated following a procedure for the synthesis of $3 \mathrm{a}$ as a white solid, mp $169.3-170.1^{\circ} \mathrm{C} .{ }^{1} \mathrm{H}$ $\operatorname{NMR}\left(400 \mathrm{MHz}, \mathrm{CDCl}_{3}\right) \delta 8.35(\mathrm{~d}, J=7.5 \mathrm{~Hz}, 1 \mathrm{H}), 8.02(\mathrm{dd}, J=8.8$, $5.3 \mathrm{~Hz}, 1 \mathrm{H}), 7.69(\mathrm{dd}, J=12.3,4.9 \mathrm{~Hz}, 2 \mathrm{H}), 7.41(\mathrm{t}, J=7.8 \mathrm{~Hz}, 3 \mathrm{H})$, $7.05(\mathrm{dd}, J=14.9,5.4 \mathrm{~Hz}, 3 \mathrm{H}), 6.97(\mathrm{dd}, J=9.7,1.8 \mathrm{~Hz}, 1 \mathrm{H}), 3.91(\mathrm{~s}$, $3 \mathrm{H}) \mathrm{ppm} .{ }^{13} \mathrm{C}$ NMR $\left(100 \mathrm{MHz}, \mathrm{CDCl}_{3}\right) \delta 168.7,165.0,162.6,159.6$, 155.5, 145.9, 140.1, 140.0, 132.9, 129.7, 128.9, 126.5, 124.5, 124.2, 121.7, 121.6, 121.5, 118.1, 114.5, 112.4, 111.0, 110.8, 98.0, 97.7, $55.7 \mathrm{ppm} .{ }^{19} \mathrm{~F}$ NMR $\left(376 \mathrm{MHz}, \mathrm{CDCl}_{3}\right) \delta-109.8,-109.8,-109.8$, $-109.8,-109.8,-109.9 \mathrm{ppm}$. HRMS (ESI) $\mathrm{m} / \mathrm{z}$ calcd for $\mathrm{C}_{22} \mathrm{H}_{15} \mathrm{FNO}_{3}[\mathrm{M}+\mathrm{H}]^{+}: 360.1030$, found: 360.1014.

7-Fluoro-10-(4-methoxyphenyl)chromeno[3,2-b]indol-11(10H)one (3e). $3 \mathbf{e}(20 \mathrm{mg}, 56 \%$ yield) was generated following a procedure for the synthesis of $3 \mathrm{a}$ as a white solid, $\mathrm{mp}$ 208.9-209.6 ${ }^{\circ} \mathrm{C} .{ }^{1} \mathrm{H}$ NMR (400 MHz, $\left.\mathrm{CDCl}_{3}\right) \delta 8.40-8.30(\mathrm{~m}, 2 \mathrm{H}), 7.53-7.47(\mathrm{~m}, 2 \mathrm{H})$, 7.46-7.41 (m, 2H), 7.40-7.35 (m, 2H), $7.32(\mathrm{~d}, J=8.4 \mathrm{~Hz}, 1 \mathrm{H}), 7.08$ (d, $J=8.9 \mathrm{~Hz}, 2 \mathrm{H}), 3.92(\mathrm{~s}, 3 \mathrm{H}) \mathrm{ppm} .{ }^{13} \mathrm{C}$ NMR $\left(100 \mathrm{MHz}, \mathrm{CDCl}_{3}\right)$ $\delta 159.7,155.0,151.2,142.5,129.5,129.2,127.7,124.6,123.6,122.7$, 122.4, 122.3, 121.8, 121.6, 118.8, 117.3, 114.5, 112.5, $55.6 \mathrm{ppm}$. HRMS (ESI) $\mathrm{m} / \mathrm{z}$ calcd for $\mathrm{C}_{22} \mathrm{H}_{15} \mathrm{FNO}_{3}[\mathrm{M}+\mathrm{H}]^{+}: 360.1030$, found: 360.1036.

10-(4-Methoxyphenyl)thiochromeno[3,2-b]indol-11(10H)one (3f). 3f (19 mg, 53\% yield) was generated following a procedure for the synthesis of $3 \mathrm{a}$ as a white solid, $\mathrm{mp} 224.9-$ $225.5^{\circ} \mathrm{C} .{ }^{1} \mathrm{H}$ NMR $\left(400 \mathrm{MHz}, \mathrm{CDCl}_{3}\right) \delta 8.64(\mathrm{~d}, J=8.1 \mathrm{~Hz}, 1 \mathrm{H})$, $7.91(\mathrm{~d}, J=8.0 \mathrm{~Hz}, 1 \mathrm{H}), 7.77(\mathrm{~d}, J=8.0 \mathrm{~Hz}, 1 \mathrm{H}), 7.65-7.56(\mathrm{~m}$, $1 \mathrm{H}), 7.48(\mathrm{dd}, J=18.4,7.5 \mathrm{~Hz}, 2 \mathrm{H}), 7.35(\mathrm{~d}, J=8.8 \mathrm{~Hz}, 2 \mathrm{H})$, $7.33-7.29(\mathrm{~m}, 1 \mathrm{H}), 7.25(\mathrm{~d}, J=9.7 \mathrm{~Hz}, 1 \mathrm{H}), 7.08(\mathrm{~d}, J=8.8 \mathrm{~Hz}$, $2 \mathrm{H}), 3.92(\mathrm{~s}, 3 \mathrm{H}) \mathrm{ppm} .{ }^{13} \mathrm{C}$ NMR $\left(100 \mathrm{MHz}, \mathrm{CDCl}_{3}\right) \delta 172.3$, $159.4,141.2,135.9,132.8,131.4,130.8,129.2,129.2,128.6$, $128.2,126.9,126.2,123.2,121.2,120.6,120.3,114.3,112.2$, $55.6 \mathrm{ppm}$. HRMS (ESI) $\mathrm{m} / z$ calcd for $\mathrm{C}_{22} \mathrm{H}_{16} \mathrm{NO}_{2} \mathrm{~S}[\mathrm{M}+\mathrm{H}]^{+}$: 358.0896, found: 358.0889 .

7-(4-Methoxyphenyl)-7H-indolo[2,3-c]quinolone $\quad(3 \mathrm{~g}) . \quad 3 \mathrm{~g}$ ( $25 \mathrm{mg}, 77 \%$ yield) was generated following a procedure for the synthesis of 3a as a white solid, mp 130.4-131.2 ${ }^{\circ} \mathrm{C} .{ }^{1} \mathrm{H}$ NMR $\left(400 \mathrm{MHz}, \mathrm{CDCl}_{3}\right) \delta 9.08(\mathrm{~s}, 1 \mathrm{H}), 8.77(\mathrm{~d}, J=8.2 \mathrm{~Hz}, 1 \mathrm{H}), 8.64(\mathrm{~d}, J$ $=8.0 \mathrm{~Hz}, 1 \mathrm{H}), 8.31(\mathrm{~d}, J=8.3 \mathrm{~Hz}, 1 \mathrm{H}), 7.76(\mathrm{t}, J=7.5 \mathrm{~Hz}, 1 \mathrm{H})$, $7.69(\mathrm{t}, J=7.6 \mathrm{~Hz}, 1 \mathrm{H}), 7.59-7.54(\mathrm{~m}, 1 \mathrm{H}), 7.48(\mathrm{dd}, J=18.5$, $7.9 \mathrm{~Hz}, 4 \mathrm{H}), 7.17(\mathrm{~d}, J=8.7 \mathrm{~Hz}, 2 \mathrm{H}), 3.95(\mathrm{~s}, 3 \mathrm{H}) \mathrm{ppm} .{ }^{13} \mathrm{C} \mathrm{NMR}$ $\left(100 \mathrm{MHz}, \mathrm{CDCl}_{3}\right) \delta 159.7,143.5,141.4,137.4,134.1,130.5$, 129.1, 128.9, 127.4, 127.2, 125.9, 124.7, 123.5, 123.4, 122.2, 121.4, 121.3, 115.5, 111.3, $55.8 \mathrm{ppm}$. HRMS (ESI) $\mathrm{m} / \mathrm{z}$ calcd for $\mathrm{C}_{22} \mathrm{H}_{17} \mathrm{~N}_{2} \mathrm{O}[\mathrm{M}+\mathrm{H}]^{+}: 325.1335$, found: 325.1330. 
7-(4-Methoxyphenyl)-9-methyl-7 $\mathrm{H}$-indolo[2,3-c]quinolone (3h). $3 \mathbf{h}$ ( $25 \mathrm{mg}, 74 \%$ yield) was generated following a procedure for the synthesis of $\mathbf{3 a}$ as a white solid, mp 177.9$179.1{ }^{\circ} \mathrm{C} .{ }^{1} \mathrm{H}$ NMR $\left(400 \mathrm{MHz}, \mathrm{CDCl}_{3}\right) \delta 9.02(\mathrm{~s}, 1 \mathrm{H}), 8.71(\mathrm{~d}, J=$ $8.1 \mathrm{~Hz}, 1 \mathrm{H}), 8.47(\mathrm{~d}, J=8.7 \mathrm{~Hz}, 1 \mathrm{H}), 8.28(\mathrm{~d}, J=8.2 \mathrm{~Hz}, 1 \mathrm{H})$, $7.72(\mathrm{t}, J=7.4 \mathrm{~Hz}, 1 \mathrm{H}), 7.65(\mathrm{t}, J=7.5 \mathrm{~Hz}, 1 \mathrm{H}), 7.47(\mathrm{~d}, J=$ $8.8 \mathrm{~Hz}, 2 \mathrm{H}), 7.26(\mathrm{t}, J=7.6 \mathrm{~Hz}, 2 \mathrm{H}), 7.15(\mathrm{~d}, J=8.8 \mathrm{~Hz}, 2 \mathrm{H})$, $3.93(\mathrm{~s}, 3 \mathrm{H}), 2.53(\mathrm{~s}, 3 \mathrm{H}) \mathrm{ppm} .{ }^{13} \mathrm{C} \mathrm{NMR}\left(100 \mathrm{MHz}, \mathrm{CDCl}_{3}\right.$ ) $\delta 159.6,143.5,141.9,137.8,137.3,134.1,130.5,129.2,128.9$, 127.1, 125.8, 124.6, 123.5, 123.1, 123.1, 121.5, 120.0, 115.4, 111.1, 55.8, 22.3 ppm. HRMS (ESI) $\mathrm{m} / z$ calcd for $\mathrm{C}_{23} \mathrm{H}_{19} \mathrm{~N}_{2} \mathrm{O}$ $[\mathrm{M}+\mathrm{H}]^{+}$: 339.1492, found: 339.1487.

9-Chloro-7-(4-methoxyphenyl)-7H-indolo[2,3-c] quinolone (3i). $3 \mathbf{i}$ (18 mg, 51\% yield) was generated following a procedure for the synthesis of $\mathbf{3 a}$ as a white solid, mp 204.7$205.6{ }^{\circ} \mathrm{C} .{ }^{1} \mathrm{H}$ NMR $\left(400 \mathrm{MHz}, \mathrm{CDCl}_{3}\right) \delta 9.02(\mathrm{~s}, 1 \mathrm{H}), 8.65(\mathrm{~d}, J=$ $8.0 \mathrm{~Hz}, 1 \mathrm{H}), 8.48$ (d, $J=8.5 \mathrm{~Hz}, 1 \mathrm{H}), 8.30(\mathrm{~d}, J=8.1 \mathrm{~Hz}, 1 \mathrm{H})$, $7.74(\mathrm{t}, J=7.4 \mathrm{~Hz}, 1 \mathrm{H}), 7.68(\mathrm{t}, J=7.4 \mathrm{~Hz}, 1 \mathrm{H}), 7.46(\mathrm{~d}, J=$ $8.5 \mathrm{~Hz}, 3 \mathrm{H}), 7.40(\mathrm{~d}, J=8.6 \mathrm{~Hz}, 1 \mathrm{H}), 7.17(\mathrm{~d}, J=8.7 \mathrm{~Hz}, 2 \mathrm{H})$, $3.95(\mathrm{~s}, 3 \mathrm{H}) \mathrm{ppm} .{ }^{13} \mathrm{C} \mathrm{NMR}\left(100 \mathrm{MHz}, \mathrm{CDCl}_{3}\right) \delta 160.0,143.3$, $141.9,137.1,134.4,133.4,130.4,128.8,128.4$, 127.6, 126.4, $124.3,123.3,122.1,121.2,120.7,115.6,111.3,55.8 \mathrm{ppm}$. HRMS (ESI) $m / z$ calcd for $\mathrm{C}_{22} \mathrm{H}_{16} \mathrm{ClN}_{2} \mathrm{O}[\mathrm{M}+\mathrm{H}]^{+}$: 359.0946, found: 359.0940 .

2-Fluoro-7-(4-methoxyphenyl)-7H-indolo[2,3-c]quinolone (3j). $3 \mathbf{j}$ ( $21 \mathrm{mg}, 62 \%$ yield) was generated following a procedure for the synthesis of $\mathbf{3 a}$ as a white solid, $\mathrm{mp}$ 161.1$161.8{ }^{\circ} \mathrm{C} .{ }^{1} \mathrm{H}$ NMR $\left(400 \mathrm{MHz}, \mathrm{CDCl}_{3}\right) \delta 9.01(\mathrm{~s}, 1 \mathrm{H}), 8.52(\mathrm{~d}, J=$ $7.9 \mathrm{~Hz}, 1 \mathrm{H}), 8.28$ (ddd, $J=15.1,9.5,4.2 \mathrm{~Hz}, 2 \mathrm{H}), 7.60-7.53(\mathrm{~m}$, $1 \mathrm{H}), 7.52-7.45(\mathrm{~m}, 4 \mathrm{H}), 7.41(\mathrm{td}, J=8.8,2.7 \mathrm{~Hz}, 1 \mathrm{H}), 7.17(\mathrm{~d}, J$ $=8.8 \mathrm{~Hz}, 2 \mathrm{H}), 3.95(\mathrm{~s}, 3 \mathrm{H}) \mathrm{ppm} .{ }^{13} \mathrm{C} \mathrm{NMR}\left(100 \mathrm{MHz}, \mathrm{CDCl}_{3}\right)$ $\delta 162.7,160.3,159.8,141.3,140.4,136.7,134.2,132.8,132.7$, $128.9,128.8,127.3,125.4,125.3,122.9,122.0,121.6,121.0$, 115.6, 115.5, 115.3, 111.4, 107.6, 107.4, $55.8 \mathrm{ppm} .{ }^{19} \mathrm{~F}$ NMR $\left(376 \mathrm{MHz}, \mathrm{CDCl}_{3}\right) \delta-112.2,-112.2,-112.2,-112.3 \mathrm{ppm}$. HRMS (ESI) $m / z$ calcd for $\mathrm{C}_{22} \mathrm{H}_{16} \mathrm{FN}_{2} \mathrm{O}[\mathrm{M}+\mathrm{H}]^{+}: 343.1241$, found: 343.1229 .

11-(4-Methoxyphenyl)-11H-indolo[3,2-c]isoquinoline (3k). 3k (25 $\mathrm{mg}, 78 \%$ yield) was generated following a procedure for the synthesis of $3 \mathrm{a}$ as a white solid, mp 185.8-186.9 ${ }^{\circ} \mathrm{C}$. ${ }^{1} \mathrm{H}$ NMR $\left(400 \mathrm{MHz}, \mathrm{CDCl}_{3}\right) \delta 9.17(\mathrm{~s}, 1 \mathrm{H}), 8.49(\mathrm{~d}, J=7.4 \mathrm{~Hz}, 1 \mathrm{H}), 8.12(\mathrm{~d}, J$ $=8.0 \mathrm{~Hz}, 1 \mathrm{H}), 7.53(\mathrm{t}, J=7.3 \mathrm{~Hz}, 1 \mathrm{H}), 7.50-7.39(\mathrm{~m}, 6 \mathrm{H}), 7.21(\mathrm{~d}$, $J=8.0 \mathrm{~Hz}, 1 \mathrm{H}), 7.16(\mathrm{~d}, J=8.7 \mathrm{~Hz}, 2 \mathrm{H}), 3.97$ (s, 3H) ppm. ${ }^{13} \mathrm{C}$ $\mathrm{NMR}\left(100 \mathrm{MHz}, \mathrm{CDCl}_{3}\right) \delta 160.2,146.2,142.0,135.1,131.6,130.2$, $129.4,129.1,128.4,127.7,126.1,125.7,124.5,122.9,121.2$, $121.0,119.9,115.4,110.4,55.8 \mathrm{ppm}$. HRMS (ESI) $\mathrm{m} / \mathrm{z}$ calcd for $\mathrm{C}_{22} \mathrm{H}_{17} \mathrm{~N}_{2} \mathrm{O}[\mathrm{M}+\mathrm{H}]^{+}:$325.1335, found: 325.1327.

11-(4-Methoxyphenyl)-9-methyl-11 $H$-indolo[3,2-c]isoquinoline (31). 31 ( $25 \mathrm{mg}, 74 \%$ yield) was generated following a procedure for the synthesis of 3a as a white solid, mp 173.8-175.2 ${ }^{\circ} \mathrm{C} .{ }^{1} \mathrm{H}$ NMR $\left(400 \mathrm{MHz}, \mathrm{CDCl}_{3}\right) \delta 9.15(\mathrm{~s}, 1 \mathrm{H}), 8.37(\mathrm{~d}, J=8.0 \mathrm{~Hz}, 1 \mathrm{H}), 8.11(\mathrm{~d}, J=$ $8.1 \mathrm{~Hz}, 1 \mathrm{H}), 7.54-7.49(\mathrm{~m}, 1 \mathrm{H}), 7.48-7.42(\mathrm{~m}, 3 \mathrm{H}), 7.39$ (d, $J=$ $8.4 \mathrm{~Hz}, 1 \mathrm{H}), 7.26-7.22(\mathrm{~m}, 1 \mathrm{H}), 7.17$ (d, $J=8.8 \mathrm{~Hz}, 2 \mathrm{H}), 6.99(\mathrm{~s}, 1 \mathrm{H})$, 3.98 (s, 3H), 2.50 (s, 3H) ppm. $\left.{ }^{13} \mathrm{C} \mathrm{NMR} \mathrm{(100} \mathrm{MHz,} \mathrm{CDCl}_{3}\right) \delta$ 160.1, 145.8, 142.5, 136.6, 135.1, 131.7, 130.3, 129.4, 129.2, 128.2, 127.5, $125.4,124.6,122.8,121.1,120.6,119.7,115.4,110.4,55.8$,
22.3 ppm. HRMS (ESI) $m / z$ calcd for $\mathrm{C}_{23} \mathrm{H}_{19} \mathrm{~N}_{2} \mathrm{O}[\mathrm{M}+\mathrm{H}]^{+}:$339.1492, found: 339.1489 .

7-(4-Methoxyphenyl)chromeno[3,4-b]indol-6(7H)-one (3m). $3 \mathrm{~m}$ ( $26 \mathrm{mg}, 75 \%$ yield) was generated following a procedure for the synthesis of $3 \mathrm{a}$ as a white solid, $\mathrm{mp} 231.1-232.5{ }^{\circ} \mathrm{C} .{ }^{1} \mathrm{H} \mathrm{NMR}$ $\left(400 \mathrm{MHz}, \mathrm{CDCl}_{3}\right) \delta 8.35(\mathrm{dd}, J=8.0,1.3 \mathrm{~Hz}, 1 \mathrm{H}), 7.76-7.64(\mathrm{~m}$, $3 \mathrm{H}), 7.44-7.35(\mathrm{~m}, 3 \mathrm{H}), 7.29-7.18(\mathrm{~m}, 3 \mathrm{H}), 7.07$ (d, $J=8.9 \mathrm{~Hz}$, $2 \mathrm{H}), 3.91(\mathrm{~s}, 3 \mathrm{H}) \mathrm{ppm} .{ }^{13} \mathrm{C} \mathrm{NMR}\left(100 \mathrm{MHz}, \mathrm{CDCl}_{3}\right) \delta 169.4,159.5$, 155.6 , 145.5, 136.0, 133.1, 129.8, 129.1, 126.4, 124.5, 124.1, $122.1,118.1,117.8,117.5,115.5,115.4,114.4,113.1,113.0$, 104.7, 104.4, 55.7 ppm. HRMS (ESI) $m / z$ calcd for $\mathrm{C}_{22} \mathrm{H}_{16} \mathrm{NO}_{3}[\mathrm{M}$ $+\mathrm{H}]^{+}: 342.1125$, found: 342.1124 .

4-(4-Methoxyphenyl)-4H-thieno[3,2-b]indole (3n). 3n (16 mg, $57 \%$ yield) was generated following a procedure for the synthesis of $3 a$ as a white solid, mp 125.1-126.9 ${ }^{\circ} \mathrm{C} .{ }^{1} \mathrm{H}$ NMR $\left(400 \mathrm{MHz}, \mathrm{CDCl}_{3}\right) \delta 7.80(\mathrm{~d}, J=7.4 \mathrm{~Hz}, 1 \mathrm{H}), 7.48(\mathrm{t}, J=8.6 \mathrm{~Hz}$, $3 \mathrm{H}), 7.36(\mathrm{~d}, J=5.2 \mathrm{~Hz}, 1 \mathrm{H}), 7.29-7.19(\mathrm{~m}, 2 \mathrm{H}), 7.08(\mathrm{~d}, J=$ $8.8 \mathrm{~Hz}, 2 \mathrm{H}), 7.04(\mathrm{~d}, J=5.2 \mathrm{~Hz}, 1 \mathrm{H}), 3.91(\mathrm{~s}, 3 \mathrm{H}) \mathrm{ppm} .{ }^{13} \mathrm{C} \mathrm{NMR}$ $\left(100 \mathrm{MHz}, \mathrm{CDCl}_{3}\right) \delta 158.5,145.6,141.8,131.8,126.9,126.8$, $124.4,123.0,122.2,120.1,119.0,117.4,115.0,114.7,111.4$, 111.0, 55.7 ppm. HRMS (ESI) $m / z$ calcd for $\mathrm{C}_{17} \mathrm{H}_{14} \mathrm{NOS}[\mathrm{M}+\mathrm{H}]^{+}$: 280.0791, found: 280.0787 .

10-Phenylchromeno[3,2-b]indol-11(10H)-one (4a). 4 a (24 mg, $79 \%$ yield) was generated following a procedure for the synthesis of $3 \mathbf{a}$ as a white solid, mp 190.7-191.6 ${ }^{\circ} \mathrm{C} .{ }^{1} \mathrm{H}$ NMR $\left(400 \mathrm{MHz}, \mathrm{CDCl}_{3}\right) \delta 8.37(\mathrm{~d}, J=7.8 \mathrm{~Hz}, 1 \mathrm{H}), 8.10(\mathrm{~d}, J=8.1 \mathrm{~Hz}$, $1 \mathrm{H}), 7.72(\mathrm{~d}, J=3.0 \mathrm{~Hz}, 2 \mathrm{H}), 7.60-7.54(\mathrm{~m}, 2 \mathrm{H}), 7.53-7.46(\mathrm{~m}$, $4 \mathrm{H}), 7.45-7.40(\mathrm{~m}, 1 \mathrm{H}), 7.38(\mathrm{~d}, J=8.5 \mathrm{~Hz}, 1 \mathrm{H}), 7.33(\mathrm{t}, J=$ $7.5 \mathrm{~Hz}, 1 \mathrm{H}) \mathrm{ppm} .{ }^{13} \mathrm{C} \mathrm{NMR}\left(100 \mathrm{MHz}, \mathrm{CDCl}_{3}\right) \delta 169.1,155.5$, 146.3, 139.0, 137.1, 132.8, 129.0, 128.7, 128.1, 128.0, 126.4, 124.5, 124.0, 121.2, 120.6, 120.0, 118.1, 115.7, 111.5 ppm. HRMS (ESI) $m / z$ calcd for $\mathrm{C}_{21} \mathrm{H}_{14} \mathrm{NO}_{2}[\mathrm{M}+\mathrm{H}]^{+}:$312.1019, found: 312.1017.

10-( $p$-Tolyl)chromeno[3,2-b]indol-11(10H)-one $\quad(4 b) . \quad 4 b$ (30 mg, 92\% yield) was generated following a procedure for the synthesis of 3a as a white solid, mp 205.2-206.6 ${ }^{\circ} \mathrm{C} .{ }^{1} \mathrm{H}$ NMR $\left(400 \mathrm{MHz}, \mathrm{CDCl}_{3}\right) \delta 8.37(\mathrm{~d}, J=7.9 \mathrm{~Hz}, 1 \mathrm{H}), 8.09(\mathrm{~d}, J=8.1 \mathrm{~Hz}$, $1 \mathrm{H}), 7.71(\mathrm{~d}, J=3.0 \mathrm{~Hz}, 2 \mathrm{H}), 7.51-7.45(\mathrm{~m}, 1 \mathrm{H}), 7.44-7.40(\mathrm{~m}$, 1H), 7.41-7.34 (m, 5H), $7.31(\mathrm{t}, J=7.5 \mathrm{~Hz}, 1 \mathrm{H}), 2.48$ (s, 3H) ppm. ${ }^{13} \mathrm{C} \mathrm{NMR}\left(100 \mathrm{MHz}, \mathrm{CDCl}_{3}\right) \delta 169.1,155.5,146.1,139.1,138.0$, $134.5,132.8,129.7,128.6,127.7,126.4,124.5,124.0,121.1$, 120.7, 120.0, 118.0, 115.6, 111.6, 21.4 ppm. HRMS (ESI) $\mathrm{m} / \mathrm{z}$ calcd for $\mathrm{C}_{22} \mathrm{H}_{16} \mathrm{NO}_{2}[\mathrm{M}+\mathrm{H}]^{+}$: 326.1176, found: 326.1171 .

10-(4-Fluorophenyl)chromeno[3,2-b]indol-11(10H)-one (4c). 4c (24 mg, 74\% yield) was generated following a procedure for the synthesis of $3 \mathrm{a}$ as a white solid, mp 227.5-228.6 ${ }^{\circ} \mathrm{C} .{ }^{1} \mathrm{H} \mathrm{NMR}$ $\left(400 \mathrm{MHz}, \mathrm{CDCl}_{3}\right) \delta 8.37(\mathrm{~d}, J=7.9 \mathrm{~Hz}, 1 \mathrm{H}), 8.09(\mathrm{~d}, J=8.1 \mathrm{~Hz}$, $1 \mathrm{H}), 7.72(\mathrm{t}, J=5.8 \mathrm{~Hz}, 2 \mathrm{H}), 7.57-7.40(\mathrm{~m}, 4 \mathrm{H}), 7.38-7.30(\mathrm{~m}$, $2 \mathrm{H}), 7.26$ (dd, $J=11.0,6.0 \mathrm{~Hz}, 2 \mathrm{H}) \mathrm{ppm} .{ }^{13} \mathrm{C} \mathrm{NMR}(100 \mathrm{MHz}$, $\left.\mathrm{CDCl}_{3}\right) \delta 169.2,163.4,161.0,155.6,146.3,139.2,133.2,133.0$, $129.8,129.7,128.9,126.3,124.5,124.2,121.4,120.7,120.2$, 118.2, 116.1, 115.9, 115.8, 111.4 ppm. ${ }^{19} \mathrm{~F}$ NMR (376 MHz, $\left.\mathrm{CDCl}_{3}\right) \delta-113.4,-113.5,-113.5,-113.5,-113.5,-113.5$, -113.5 ppm. HRMS (ESI) $m / z$ calcd for $\mathrm{C}_{21} \mathrm{H}_{13} \mathrm{FNO}_{2}[\mathrm{M}+\mathrm{H}]^{+}$: 330.0925, found: 330.0934 .

10-(4-Chlorophenyl)chromeno[3,2-b]indol-11(10H)-one (4d). 4d (23 mg, 65\% yield) was generated following a procedure for 
the synthesis of $3 \mathrm{a}$ as a white solid, mp $265.6-266.2{ }^{\circ} \mathrm{C} .{ }^{1} \mathrm{H}$ NMR $\left(400 \mathrm{MHz}, \mathrm{CDCl}_{3}\right) \delta 8.40-8.30(\mathrm{~m}, 1 \mathrm{H}), 8.09(\mathrm{~d}, J=8.0 \mathrm{~Hz}, 1 \mathrm{H})$, $7.72(\mathrm{dd}, J=5.7,1.4 \mathrm{~Hz}, 2 \mathrm{H}), 7.53(\mathrm{~d}, J=8.6 \mathrm{~Hz}, 2 \mathrm{H}), 7.51-7.48$ (m, 1H), 7.47-7.40 (m, 3H), 7.39-7.31 (m, 2H) ppm. ${ }^{13} \mathrm{C}$ NMR $\left(100 \mathrm{MHz}, \mathrm{CDCl}_{3}\right) \delta 169.2,155.6,146.5,139.0,135.7,133.9$, $133.1,129.3,129.0,126.4,124.5$, 124.3, 121.6, 120.6, 120.2, 118.2, 116.0, 111.4 ppm. HRMS (ESI) $m / z$ calcd for $\mathrm{C}_{21} \mathrm{H}_{13} \mathrm{ClNO}_{2}$ $[\mathrm{M}+\mathrm{H}]^{+}:$346.0629, found: 346.0621 .

10-(4-Bromophenyl)chromeno[3,2-b]indol-11(10H)-one (4e). 4e (22 mg, 65\% yield) was generated following a procedure for the synthesis of $3 \mathrm{a}$ as a white solid, $\mathrm{mp} 283.5-284.7^{\circ} \mathrm{C} .{ }^{1} \mathrm{H}$ NMR $\left(400 \mathrm{MHz}, \mathrm{CDCl}_{3}\right) \delta 8.36(\mathrm{~d}, J=8.0 \mathrm{~Hz}, 1 \mathrm{H}), 8.09(\mathrm{~d}, J=8.0 \mathrm{~Hz}$, $1 \mathrm{H}), 7.78-7.64(\mathrm{~m}, 4 \mathrm{H}), 7.50(\mathrm{t}, J=7.7 \mathrm{~Hz}, 1 \mathrm{H}), 7.46-7.29(\mathrm{~m}$, $5 \mathrm{H}) \mathrm{ppm} .{ }^{13} \mathrm{C}$ NMR $\left(100 \mathrm{MHz}, \mathrm{CDCl}_{3}\right) \delta 169.2,155.6,146.5$, $138.9,136.2$, 133.1, 132.3, 129.6, 129.1, 126.4, 124.5, 124.3, 121.9, 121.6, 120.5, 120.3, 118.2, 116.1, 111.4 ppm. HRMS (ESI) $m / z$ calcd for $\mathrm{C}_{21} \mathrm{H}_{13} \mathrm{BrNO}_{2}[\mathrm{M}+\mathrm{H}]^{+}: 390.0124$, found: 390.0127 .

10-(4-Iodophenyl)chromeno[3,2-b]indol-11(10H)-one (4f). 4f (24 mg, 54\% yield) was generated following a procedure for the synthesis of 3a as a white solid, mp 241.1-242.4 ${ }^{\circ} \mathrm{C}$. ${ }^{1} \mathrm{H}$ NMR $\left(400 \mathrm{MHz}, \mathrm{CDCl}_{3}\right) \delta 8.36(\mathrm{~d}, J=7.7 \mathrm{~Hz}, 1 \mathrm{H}), 8.10(\mathrm{~d}, J=8.0 \mathrm{~Hz}$, $1 \mathrm{H}), 7.88(\mathrm{~d}, J=8.4 \mathrm{~Hz}, 2 \mathrm{H}), 7.72(\mathrm{t}, J=6.1 \mathrm{~Hz}, 2 \mathrm{H}), 7.54-7.48$ (m, 1H), 7.46-7.41 (m, 1H), 7.35 (dd, $J=17.3,8.1 \mathrm{~Hz}, 2 \mathrm{H}), 7.27$ $(\mathrm{d}, J=6.7 \mathrm{~Hz}, 2 \mathrm{H}) \mathrm{ppm} .{ }^{13} \mathrm{C} \mathrm{NMR}\left(100 \mathrm{MHz}, \mathrm{CDCl}_{3}\right) \delta 169.1$, 155.5, 146.6, 138.8, 138.2, 136.9, 133.0, 129.8, 129.0, 126.4, 124.5, 124.3, 121.6, 120.5, 120.3, 118.2, 116.1, 111.4, 93.3 ppm. HRMS (ESI) $m / z$ calcd for $\mathrm{C}_{21} \mathrm{H}_{13} \mathrm{INO}_{2}[\mathrm{M}+\mathrm{H}]^{+}: 437.9986$, found: 437.9986.

10-(4-(Trifluoromethyl)phenyl)chromeno[3,2-b]indol-11(10H)one (4g). $4 \mathrm{~g}$ (20 mg, 52\% yield) was generated following a procedure for the synthesis of $3 \mathbf{a}$ as a white solid, mp $247.8-248.5^{\circ} \mathrm{C} .{ }^{1} \mathrm{H}$ NMR (400 MHz, $\left.\mathrm{CDCl}_{3}\right) \delta 8.40-8.33(\mathrm{~m}, 1 \mathrm{H}), 8.12(\mathrm{~d}, J=8.1 \mathrm{~Hz}$, $1 \mathrm{H}), 7.83(\mathrm{~d}, J=8.4 \mathrm{~Hz}, 2 \mathrm{H}), 7.74(\mathrm{dt}, J=8.0,4.0 \mathrm{~Hz}, 2 \mathrm{H}), 7.65(\mathrm{~d}, J$ $=8.3 \mathrm{~Hz}, 2 \mathrm{H}), 7.52(\mathrm{dd}, J=11.4,4.1 \mathrm{~Hz}, 1 \mathrm{H}), 7.47-7.43(\mathrm{~m}, 1 \mathrm{H})$, 7.43-7.34 (m, 2H) ppm. $\left.{ }^{13} \mathrm{C} \mathrm{NMR} \mathrm{(100} \mathrm{MHz,} \mathrm{CDCl}_{3}\right) \delta 169.2,155.5$, 147.0, 140.2, 138.8, 133.2, 129.3, 128.3, 126.4, 126.2, 124.4, 121.9, 120.4, 118.2, 116.3, 111.3 ppm. ${ }^{19} \mathrm{~F}$ NMR (376 MHz, $\mathrm{CDCl}_{3}$ ) $\delta-62.4 \mathrm{ppm}$. HRMS (ESI) $\mathrm{m} / z$ calcd for $\mathrm{C}_{22} \mathrm{H}_{13} \mathrm{~F}_{3} \mathrm{NO}_{2}[\mathrm{M}+\mathrm{H}]^{+}$: 380.0893, found: 380.0908 .

Methyl 4-(11-oxochromeno[3,2-b]indol-10(11H)-yl)benzoate (4h). $4 \mathrm{~h}$ (15 mg, 41\% yield) was generated following a procedure for the synthesis of $3 \mathrm{a}$ as a white solid, mp $232.4-233.1{ }^{\circ} \mathrm{C} .{ }^{1} \mathrm{H}$ NMR (400 MHz, $\left.\mathrm{CDCl}_{3}\right) \delta 8.37(\mathrm{~d}, J=7.5 \mathrm{~Hz}, 1 \mathrm{H}), 8.24(\mathrm{~d}, J=$ $8.5 \mathrm{~Hz}, 2 \mathrm{H}), 8.11(\mathrm{~d}, J=8.0 \mathrm{~Hz}, 1 \mathrm{H}), 7.78-7.70(\mathrm{~m}, 2 \mathrm{H}), 7.60(\mathrm{~d}, J$ $=8.5 \mathrm{~Hz}, 2 \mathrm{H}), 7.55-7.49(\mathrm{~m}, 1 \mathrm{H}), 7.48-7.40(\mathrm{~m}, 2 \mathrm{H}), 7.36(\mathrm{t}, J=$ $7.5 \mathrm{~Hz}, 1 \mathrm{H}), 3.98(\mathrm{~s}, 3 \mathrm{H}) \mathrm{ppm} .{ }^{13} \mathrm{C} \mathrm{NMR}\left(100 \mathrm{MHz}, \mathrm{CDCl}_{3}\right)$ $\delta$ 169.1, 166.6, 155.6, 147.0, 141.2, 138.8, 133.1, 130.5, 129.5, $129.2,127.8,126.5,124.5,124.3,121.8,120.5,120.3,118.2$, $116.3,111.5,52.4$ ppm. HRMS (ESI) $m / z$ calcd for $\mathrm{C}_{23} \mathrm{H}_{16} \mathrm{NO}_{4}[\mathrm{M}$ $+\mathrm{H}]^{+}$: 370.1074, found: 370.1074 .

Methyl 2-(11-oxochromeno[3,2-b]indol-10(11H)-yl)benzoate (4i). $4 \mathbf{i}$ (12 mg, 32\% yield) was generated following a procedure for the synthesis of 3a as a white solid, mp 219.9-221.1 ${ }^{\circ} \mathrm{C} .{ }^{1} \mathrm{H}$ NMR $\left(400 \mathrm{MHz}, \mathrm{CDCl}_{3}\right) \delta 8.33(\mathrm{~d}, J=7.9 \mathrm{~Hz}, 1 \mathrm{H}), 8.20(\mathrm{dd}, J=$ $7.8,1.4 \mathrm{~Hz}, 1 \mathrm{H}), 8.10(\mathrm{~d}, J=8.0 \mathrm{~Hz}, 1 \mathrm{H}), 7.78-7.66(\mathrm{~m}, 3 \mathrm{H}), 7.61$ $(\mathrm{td}, J=7.7,1.1 \mathrm{~Hz}, 1 \mathrm{H}), 7.57(\mathrm{~d}, J=7.8 \mathrm{~Hz}, 1 \mathrm{H}), 7.50-7.43(\mathrm{~m}$, $1 \mathrm{H}), 7.43-7.37(\mathrm{~m}, 1 \mathrm{H}), 7.31(\mathrm{t}, J=7.5 \mathrm{~Hz}, 1 \mathrm{H}), 7.15(\mathrm{~d}, J=$
$8.5 \mathrm{~Hz}, 1 \mathrm{H}), 3.46(\mathrm{~s}, 3 \mathrm{H}) \mathrm{ppm} .{ }^{13} \mathrm{C} \mathrm{NMR}\left(100 \mathrm{MHz}, \mathrm{CDCl}_{3}\right)$ $\delta 169.3,165.6,155.7,146.0,139.2$, 137.3, 132.9, 132.8, 131.6, $130.5,129.5,128.8,128.7,126.3,124.4,124.0,121.5,121.2$, $120.1,118.2,115.8,111.1,52.2 \mathrm{ppm}$. HRMS (ESI) $\mathrm{m} / \mathrm{z}$ calcd for $\mathrm{C}_{23} \mathrm{H}_{16} \mathrm{NO}_{4}[\mathrm{M}+\mathrm{H}]^{+}: 370.1074$, found: 370.1078 .

10-(Pyridin-2-yl)chromeno[3,2-b]indol-11(10H)-one $(4 \mathbf{j}) . \quad 4 \mathbf{j}$ (11 mg, 34\% yield) was generated following a procedure for the synthesis of 3a as a white solid, mp 191.3-192.5 ${ }^{\circ} \mathrm{C} .{ }^{1} \mathrm{H}$ NMR $\left(400 \mathrm{MHz}, \mathrm{CDCl}_{3}\right) \delta 8.67(\mathrm{~s}, 1 \mathrm{H}), 8.39(\mathrm{~d}, J=7.9 \mathrm{~Hz}, 1 \mathrm{H}), 8.09(\mathrm{~d}, J$ $=8.0 \mathrm{~Hz}, 1 \mathrm{H}), 7.93(\mathrm{t}, J=7.2 \mathrm{~Hz}, 1 \mathrm{H}), 7.84(\mathrm{~d}, J=8.4 \mathrm{~Hz}, 1 \mathrm{H})$, $7.72(\mathrm{~s}, 2 \mathrm{H}), 7.54(\mathrm{t}, J=7.6 \mathrm{~Hz}, 2 \mathrm{H}), 7.45(\mathrm{dd}, J=14.0,6.4 \mathrm{~Hz}$, $1 \mathrm{H}), 7.42-7.33(\mathrm{~m}, 2 \mathrm{H}) \mathrm{ppm} .{ }^{13} \mathrm{C} \mathrm{NMR}\left(100 \mathrm{MHz}, \mathrm{CDCl}_{3}\right) \delta 169.0$, 155.5 , 150.6, 148.4, 147.7, 138.8, 137.9, 133.0, 129.5, 126.5, $124.6,124.3,122.6,122.5,122.1,120.1,120.0$, 118.2, 116.7, 113.0 ppm. HRMS (ESI) $m / z$ calcd for $\mathrm{C}_{20} \mathrm{H}_{13} \mathrm{~N}_{2} \mathrm{O}_{2}[\mathrm{M}+\mathrm{H}]^{+}$: 313.0972, found: 313.0963.

10-(Quinolin-8-yl)chromeno[3,2-b]indol-11(10H)-one (4k). 4k (24 mg, 67\% yield) was generated following a procedure for the synthesis of $3 \mathrm{a}$ as a white solid, mp 213.5-214.5 ${ }^{\circ} \mathrm{C} .{ }^{1} \mathrm{H}$ NMR $\left(400 \mathrm{MHz}, \mathrm{CDCl}_{3}\right) \delta 8.76(\mathrm{~d}, J=3.0 \mathrm{~Hz}, 1 \mathrm{H}), 8.28(\mathrm{dd}, J=18.7$, $7.9 \mathrm{~Hz}, 2 \mathrm{H}), 8.15(\mathrm{~d}, J=8.0 \mathrm{~Hz}, 1 \mathrm{H}), 8.03(\mathrm{~d}, J=8.2 \mathrm{~Hz}, 1 \mathrm{H}), 7.91$ $(\mathrm{d}, J=7.1 \mathrm{~Hz}, 1 \mathrm{H}), 7.73(\mathrm{dd}, J=15.9,8.4 \mathrm{~Hz}, 3 \mathrm{H}), 7.48-7.30(\mathrm{~m}$, $4 \mathrm{H}), 7.08$ (d, $J=8.4 \mathrm{~Hz}, 1 \mathrm{H}) \mathrm{ppm} .{ }^{13} \mathrm{C} \mathrm{NMR}\left(100 \mathrm{MHz}, \mathrm{CDCl}_{3}\right.$ ) $\delta 169.3,155.8,150.9,146.2,145.2,139.9,136.7,135.1,132.7$, $129.8,129.3,129.0,128.5,126.3,126.3,124.7,123.9,122.6$, 121.9, 121.2, 120.2, 118.2, 116.2, $111.8 \mathrm{ppm}$. HRMS (ESI) $\mathrm{m} / \mathrm{z}$ calcd for $\mathrm{C}_{24} \mathrm{H}_{15} \mathrm{~N}_{2} \mathrm{O}_{2}[\mathrm{M}+\mathrm{H}]^{+}$: 363.1128, found: 363.1113.

\section{General procedure B for the synthesis of 4l-4o}

The synthesis of 10-(2-(pyridin-2-yl)ethyl)chromeno[3,2- $b]$ indol$11(10 H)$-one $(4 \mathbf{l})$ is exemplified. To a stirred solution of iodonium 1a $(0.1 \mathrm{mmol})$ in $i$-PrOH $(0.9 \mathrm{~mL})$, was added ethylene glycol (0.1 mL), 2-(pyridin-2-yl)ethan-1-amine (2.5 equiv.), $\mathrm{Na}_{2} \mathrm{CO}_{3}$ (3 equiv.), $\mathrm{Cu}(\mathrm{OAc})_{2}$ ( 0.1 equiv.), and $\mathrm{CuI}$ ( 0.1 equiv.). The reaction proceeded at reflux for $16 \mathrm{~h}$ under argon atmosphere before $i$-PrOH was removed by a rotary evaporation. The remained mixture was extracted with EtOAc. The combined organic layers were washed with $\mathrm{H}_{2} \mathrm{O}$ and brine, dried over anhydrous $\mathrm{Na}_{2} \mathrm{SO}_{4}$, and evaporated in a vacuo. The residue was purified by column chromatography $(\mathrm{PE} /$ EtOAc $=15 / 1-5 / 1)$ to provide 41 as a white solid (23 $\mathrm{mg}, 68 \%$ yield), $\mathrm{mp} 139.7-$ $140.8{ }^{\circ} \mathrm{C} .{ }^{1} \mathrm{H}$ NMR $\left(400 \mathrm{MHz}, \mathrm{CDCl}_{3}\right) \delta 8.55(\mathrm{~d}, J=4.3 \mathrm{~Hz}, 1 \mathrm{H})$, $8.45(\mathrm{~d}, J=7.9 \mathrm{~Hz}, 1 \mathrm{H}), 7.95(\mathrm{~d}, J=8.0 \mathrm{~Hz}, 1 \mathrm{H}), 7.75-7.59(\mathrm{~m}$, $2 \mathrm{H}), 7.43(\mathrm{t}, J=7.5 \mathrm{~Hz}, 2 \mathrm{H}), 7.34(\mathrm{dd}, J=20.0,7.8 \mathrm{~Hz}, 2 \mathrm{H}), 7.17$ $(\mathrm{t}, J=7.4 \mathrm{~Hz}, 1 \mathrm{H}), 7.06(\mathrm{t}, J=8.4 \mathrm{~Hz}, 2 \mathrm{H}), 5.08(\mathrm{t}, J=7.3 \mathrm{~Hz}, 2 \mathrm{H})$, $3.37(\mathrm{t}, J=7.2 \mathrm{~Hz}, 2 \mathrm{H}) \mathrm{ppm} .{ }^{13} \mathrm{C} \mathrm{NMR}(100 \mathrm{MHz}, \mathrm{CDCl} 3) \delta 170.1$, $158.8,155.6,149.5,145.2$, 138.0, 136.5, 132.7, 128.1, 126.1, $124.4,123.9,123.9,121.7,120.2$, 120.0, 119.9, 118.1, 114.7, 110.5, 45.0, 39.9 ppm. HRMS (ESI) $m / z$ calcd for $\mathrm{C}_{22} \mathrm{H}_{17} \mathrm{~N}_{2} \mathrm{O}_{2}[\mathrm{M}$ $+\mathrm{H}]^{+}$: 341.1285 , found: 341.1278 .

10-Butylchromeno[3,2-b]indol-11(10H)-one (4m). 4m (20 mg, $68 \%$ yield) was generated following a procedure for the synthesis of 41 as a white solid, mp 87.4-88.6 ${ }^{\circ} \mathrm{C}$. ${ }^{1} \mathrm{H}$ NMR (400 $\left.\mathrm{MHz} \mathrm{CDCl}_{3}\right) \delta 8.45(\mathrm{dd}, J=8.0,1.3 \mathrm{~Hz}, 1 \mathrm{H}), 8.02(\mathrm{~d}, J=8.1 \mathrm{~Hz}$, $1 \mathrm{H}), 7.74-7.61(\mathrm{~m}, 2 \mathrm{H}), 7.57-7.46(\mathrm{~m}, 2 \mathrm{H}), 7.45-7.40(\mathrm{~m}, 1 \mathrm{H})$, 7.25 (ddd, $J=7.9,5.3,1.1 \mathrm{~Hz}, 1 \mathrm{H}), 4.76$ (t, $J=7.3 \mathrm{~Hz}, 2 \mathrm{H}), 1.94-$ 
$1.76(\mathrm{~m}, 2 \mathrm{H}), 1.41(\mathrm{dd}, J=15.3,7.5 \mathrm{~Hz}, 2 \mathrm{H}), 0.95(\mathrm{t}, J=7.4 \mathrm{~Hz}$, $3 \mathrm{H}) \mathrm{ppm} .{ }^{13} \mathrm{C} \mathrm{NMR}\left(100 \mathrm{MHz}, \mathrm{CDCl}_{3}\right) \delta 170.3,155.6,145.0$, $137.9,132.7,128.1,126.3,124.5,123.9,120.4,120.2,118.2$, 115.0, 110.6, 44.8, 33.1, 20.2, $14.0 \mathrm{ppm}$. HRMS (ESI) $\mathrm{m} / \mathrm{z}$ calcd for $\mathrm{C}_{19} \mathrm{H}_{18} \mathrm{NO}_{2}[\mathrm{M}+\mathrm{H}]^{+}:$292.1332, found: 292.1337.

10-Cyclopropylchromeno[3,2-b]indol-11(10H)-one (4n). 4n (23 mg, 82\% yield) was generated following a procedure for the synthesis of $4 \mathrm{l}$ as a white solid, mp 167.9-168.4 ${ }^{\circ} \mathrm{C} .{ }^{1} \mathrm{H}$ NMR $(400$ $\left.\mathrm{MHz}, \mathrm{CDCl}_{3}\right) \delta 8.46(\mathrm{dd}, J=8.0,1.4 \mathrm{~Hz}, 1 \mathrm{H}), 8.03-7.98(\mathrm{~m}, 1 \mathrm{H})$, $7.73(\mathrm{~d}, J=8.5 \mathrm{~Hz}, 1 \mathrm{H}), 7.71-7.63(\mathrm{~m}, 2 \mathrm{H}), 7.52$ (ddd, $J=8.4,5.4$, $1.2 \mathrm{~Hz}, 1 \mathrm{H}), 7.43$ (ddd, $J=8.1,6.8,1.4 \mathrm{~Hz}, 1 \mathrm{H}), 7.30-7.23(\mathrm{~m}$, $1 \mathrm{H}), 3.63-3.54(\mathrm{~m}, 1 \mathrm{H}), 1.40-1.33(\mathrm{~m}, 2 \mathrm{H}), 1.17$ (qd, $J=5.6$, $4.5 \mathrm{~Hz}, 2 \mathrm{H}) \mathrm{ppm} .{ }^{13} \mathrm{C}$ NMR $\left(100 \mathrm{MHz}, \mathrm{CDCl}_{3}\right) \delta 169.5,155.4$, $145.5,139.1,132.7,128.1,126.4,124.8,124.0$, 121.6, 120.6, $120.1,118.0,115.2,112.3,26.6,9.5$ ppm. HRMS (ESI) $\mathrm{m} / z$ calcd for $\mathrm{C}_{18} \mathrm{H}_{14} \mathrm{NO}_{2}[\mathrm{M}+\mathrm{H}]^{+}$: 276.1019, found: 276.1011.

10-(4-Hydroxybutyl)chromeno[3,2-b]indol-11(10H)-one (4o). 40 (27 mg, 88\% yield) was generated following a procedure for the synthesis of $4 \mathbf{l}$ as a white solid, mp 110.7-111.3 ${ }^{\circ} \mathrm{C} .{ }^{1} \mathrm{H}$ NMR $\left(400 \mathrm{MHz}, \mathrm{CDCl}_{3}\right) \delta 8.44(\mathrm{~d}, J=7.8 \mathrm{~Hz}, 1 \mathrm{H}), 8.03(\mathrm{~d}, J=8.1 \mathrm{~Hz}$, 1H), 7.76-7.62 (m, 2H), 7.57-7.46 (m, 2H), $7.44(\mathrm{t}, J=7.2 \mathrm{~Hz}$, $1 \mathrm{H}), 7.30-7.18(\mathrm{~m}, 1 \mathrm{H}), 4.81-4.73(\mathrm{~m}, 2 \mathrm{H}), 3.75(\mathrm{t}, J=6.2 \mathrm{~Hz}$, $2 \mathrm{H}$ ), 2.01 (dt, $J=14.8,7.4 \mathrm{~Hz}, 2 \mathrm{H}), 1.74-1.55$ (m, $2 \mathrm{H}) \mathrm{ppm} .{ }^{13} \mathrm{C}$ NMR (100 MHz, $\left.\mathrm{CDCl}_{3}\right) \delta 170.3,155.5,145.2,137.9,132.8,128.4$, 126.2, 124.2, 123.9, 120.3, 120.2, 118.1, 114.9, 110.4, 62.1, 44.2, 29.4, $27.2 \mathrm{ppm}$. HRMS (ESI) $m / z$ calcd for $\mathrm{C}_{19} \mathrm{H}_{18} \mathrm{NO}_{3}[\mathrm{M}+\mathrm{H}]^{+}$: 308.1281, found: 308.1278.

Procedure for the synthesis of triethylammonium benzylcarbamodithioate (M1). To a solution of benzylamine $(1.11 \mathrm{~g}$, $10.38 \mathrm{mmol}, 1.05$ equiv.) and $\mathrm{Et}_{3} \mathrm{~N}(1.0 \mathrm{~g}, 9.88 \mathrm{mmol}, 1$ equiv.) in $\mathrm{CH}_{2} \mathrm{Cl}_{2}$ (25 mL), $\mathrm{CS}_{2}(0.83 \mathrm{~g}, 10.87 \mathrm{mmol}, 1.1$ equiv.) was dropped slowly. The solution was stirred at room temperature for $3 \mathrm{~h}$, concentrated by a rotary evaporator, and finally dried by a high vacuum to give M1 (2.75 g, 98\% yield) as a white solid.

The reaction procedure of heterocyclic iodoniums and M1 to provide benzothiophene-fused heterocycles 5 . Syntheses of 8methyl-11 $H$-benzo[4,5]thieno[3,2- $b]$ chromen-11-one (5a) is exemplified. To a stirred solution of iodonium $\mathbf{1 b}(0.1 \mathrm{mmol})$ in MeCN (2.0 mL), was added M1 (2 equiv.) and $\mathrm{CuSO}_{4}$ (0.1 equiv.). The reaction proceeded at $70{ }^{\circ} \mathrm{C}$ for $6 \mathrm{~h}$ under argon atmosphere before MeCN was removed by a rotary evaporation. The remained mixture was extracted with EtOAc. The combined organic layers were washed with $\mathrm{H}_{2} \mathrm{O}$ and brine, dried over anhydrous $\mathrm{Na}_{2} \mathrm{SO}_{4}$, and evaporated in a vacuo. The residue was purified by column chromatography $(\mathrm{PE} / \mathrm{EtOAc}=20 / 1-5 / 1)$ to provide 5a as a white solid (22 $\mathrm{mg}, 85 \%$ yield), $\mathrm{mp} 191.2-$ $192.1{ }^{\circ} \mathrm{C} .{ }^{1} \mathrm{H}$ NMR $\left(400 \mathrm{MHz}, \mathrm{CDCl}_{3}\right) \delta 8.39(\mathrm{dd}, J=8.0,1.5 \mathrm{~Hz}$, $1 \mathrm{H}), 8.07$ (d, $J=8.2 \mathrm{~Hz}, 1 \mathrm{H}), 7.79-7.72(\mathrm{~m}, 1 \mathrm{H}), 7.72-7.64(\mathrm{~m}$, 2H), $7.48(\mathrm{dd}, J=11.0,3.9 \mathrm{~Hz}, 1 \mathrm{H}), 7.35(\mathrm{~d}, J=8.2 \mathrm{~Hz}, 1 \mathrm{H}), 2.54$ $(\mathrm{s}, 3 \mathrm{H}) \mathrm{ppm} .{ }^{13} \mathrm{C} \mathrm{NMR}\left(100 \mathrm{MHz}, \mathrm{CDCl}_{3}\right) \delta 173.3,156.0,153.9$, $140.4,140.2$, 133.7, 127.0, 126.0, 124.9, 123.6, 122.7, 122.1, 119.9, 118.1, 22.1 ppm. HRMS (ESI) $m / z$ calcd for $\mathrm{C}_{16} \mathrm{H}_{11} \mathrm{O}_{2} \mathrm{~S}[\mathrm{M}+$ $\mathrm{H}]^{+}:$267.0474, found: 267.0481 .

Benzo[4,5]thieno[2,3-c]quinolone (5b). $5 \mathbf{b}$ (18 mg, 76\% yield) was generated following a procedure for the synthesis of $\mathbf{5 a}$ as a white solid, mp 129.7-131.2 ${ }^{\circ} \mathrm{C} .{ }^{1} \mathrm{H}$ NMR (400 MHz, $\mathrm{CDCl}_{3}$ ) $\delta 9.40(\mathrm{~s}, 1 \mathrm{H}), 8.98-8.93(\mathrm{~m}, 1 \mathrm{H}), 8.91(\mathrm{dd}, J=5.8,2.9 \mathrm{~Hz}, 1 \mathrm{H})$,
8.49-8.38 (m, $1 \mathrm{H}), 8.10(\mathrm{dd}, J=5.5,3.6 \mathrm{~Hz}, 1 \mathrm{H}), 7.82(\mathrm{dd}, J=5.7$, $3.9 \mathrm{~Hz}, 2 \mathrm{H}), 7.75-7.63$ (m, 2H) ppm. ${ }^{13} \mathrm{C} \mathrm{NMR}\left(100 \mathrm{MHz}, \mathrm{CDCl}_{3}\right)$ $\delta$ 145.5, 145.4, 141.5, 135.6, 135.2, 133.4, 130.7, 127.9, 127.7, $126.1,125.5,123.9,123.0 \mathrm{ppm}$. HRMS (ESI) $\mathrm{m} / \mathrm{z}$ calcd for $\mathrm{C}_{15} \mathrm{H}_{10} \mathrm{NS}[\mathrm{M}+\mathrm{H}]^{+}: 236.0528$, found: 236.0520 .

9-Methylbenzo[4,5]thieno[3,2-c]isoquinoline (5c). 5c (15 mg, $59 \%$ yield) was generated following a procedure for the synthesis of 5a as a white solid, mp 108.8-110.3 ${ }^{\circ} \mathrm{C} .{ }^{1} \mathrm{H}$ NMR $\left(400 \mathrm{MHz}, \mathrm{CDCl}_{3}\right) \delta 9.31(\mathrm{~s}, 1 \mathrm{H}), 8.53(\mathrm{~d}, J=8.1 \mathrm{~Hz}, 1 \mathrm{H}), 8.16(\mathrm{~d}, J$ $=8.1 \mathrm{~Hz}, 1 \mathrm{H}), 8.09(\mathrm{~d}, J=8.3 \mathrm{~Hz}, 1 \mathrm{H}), 7.85(\mathrm{t}, J=7.6 \mathrm{~Hz}, 1 \mathrm{H})$, $7.76(\mathrm{~s}, 1 \mathrm{H}), 7.69(\mathrm{t}, J=7.6 \mathrm{~Hz}, 1 \mathrm{H}), 7.42(\mathrm{~d}, J=8.2 \mathrm{~Hz}, 1 \mathrm{H}), 2.57$ (s, 3H) ppm. ${ }^{13} \mathrm{C}$ NMR (100 MHz, $\left.\mathrm{CDCl}_{3}\right) \delta$ 150.2, 138.7, 137.9, 133.5 , 132.0, 131.2, 129.5, 129.1, 127.9, 127.3, 127.2, 126.9, 126.8, 123.7, 123.6, 123.0, 122.8, 122.7, 122.4, 22.0 ppm. HRMS (ESI) $m / z$ calcd for $\mathrm{C}_{16} \mathrm{H}_{12} \mathrm{NS}[\mathrm{M}+\mathrm{H}]^{+}: 250.0685$, found: 250.0677 .

6H-Benzo[4,5]thieno[2,3-c]chromen-6-one (5d). 5d (21 mg, $84 \%$ yield) was generated following a procedure for the synthesis of 5a as a white solid, mp 205.0-206.8 ${ }^{\circ} \mathrm{C} .{ }^{1} \mathrm{H}$ NMR $\left(400 \mathrm{MHz}, \mathrm{CDCl}_{3}\right) \delta 8.69-8.59(\mathrm{~m}, 1 \mathrm{H}), 8.50(\mathrm{~d}, J=8.0 \mathrm{~Hz}, 1 \mathrm{H})$, 8.06-7.97 (m, 1H), 7.67-7.60 (m, 2H), $7.56(\mathrm{q}, J=8.3 \mathrm{~Hz}, 2 \mathrm{H})$, $7.46(\mathrm{t}, J=7.2 \mathrm{~Hz}, 1 \mathrm{H}) \mathrm{ppm} .{ }^{13} \mathrm{C} \mathrm{NMR}\left(100 \mathrm{MHz}, \mathrm{CDCl}_{3}\right) \delta 158.0$, 152.6 , 143.6, 138.6, 134.9, 130.0, 128.4, 126.0, 125.6, 124.8, $124.0,123.5,118.3,118.0 \mathrm{ppm}$. HRMS (ESI) $\mathrm{m} / \mathrm{z}$ calcd for $\mathrm{C}_{15} \mathrm{H}_{9} \mathrm{O}_{2} \mathrm{~S}[\mathrm{M}+\mathrm{H}]^{+}:$253.0318, found: 253.0313 .

\section{General procedure for synthesis of 6}

The synthesis of 6-methyl-14H-dibenzo[a,c]xanthen-14-one (6a) is exemplified. To a stirred solution of 2-chlorobenzoic acid (0.1 $\mathrm{mmol})$ in 1-methyl-2-pyrrolidinone $(1.5 \mathrm{~mL})$, was added heterocyclic iodonium (1.2 equiv.), $\mathrm{Pd}(\mathrm{OAc})_{2}$ (0.1 equiv.), and $\mathrm{K}_{2} \mathrm{CO}_{3}$ (2.2 equiv.). The reaction mixture was sealed in a tube. The reaction proceeded at $140{ }^{\circ} \mathrm{C}$ for $16 \mathrm{~h}$ before it was cooled to rt. The reaction mixture was extracted with EtOAc. The combined organic layers were washed with $\mathrm{H}_{2} \mathrm{O}$ and brine, dried over anhydrous $\mathrm{Na}_{2} \mathrm{SO}_{4}$, and evaporated in a vacuo. The residue was purified by column chromatography $(\mathrm{PE} / \mathrm{EtOAc}=$ 20/1-5/1) to provide $6 \mathbf{a}$ as a white solid (20 mg, 63\% yield), mp 207.5-209.2 ${ }^{\circ} \mathrm{C} .{ }^{1} \mathrm{H}$ NMR (400 MHz, $\mathrm{CDCl}_{3}$ ) $\delta 10.08$ (dd, $J=8.4$, $0.9 \mathrm{~Hz}, 1 \mathrm{H}), 8.49$ (d, $J=8.1 \mathrm{~Hz}, 1 \mathrm{H}), 8.39(\mathrm{dd}, J=8.0,1.8 \mathrm{~Hz}$, $2 \mathrm{H}), 8.26(\mathrm{~s}, 1 \mathrm{H}), 7.69$ (dddd, $J=12.7,8.3,7.1,1.4 \mathrm{~Hz}, 2 \mathrm{H}), 7.62-$ $7.56(\mathrm{~m}, 1 \mathrm{H}), 7.54(\mathrm{~d}, J=8.2 \mathrm{~Hz}, 1 \mathrm{H}), 7.45-7.40(\mathrm{~m}, 1 \mathrm{H}), 7.38(\mathrm{~d}, J$ $=8.3 \mathrm{~Hz}, 1 \mathrm{H}), 2.55(\mathrm{~s}, 3 \mathrm{H}) \mathrm{ppm} .{ }^{13} \mathrm{C} \mathrm{NMR}\left(100 \mathrm{MHz}, \mathrm{CDCl}_{3}\right)$ $\delta 178.2,155.3,154.3,141.0,133.9,133.6,129.2,128.9,128.4$, $127.7,127.2$, 126.7, 126.5, 124.6, 124.0, 123.9, 122.7, 122.3, 121.6, 117.5, 112.0, 22.4 ppm. HRMS (ESI) $\mathrm{m} / \mathrm{z}$ calcd for $\mathrm{C}_{22} \mathrm{H}_{15} \mathrm{O}_{2}[\mathrm{M}+\mathrm{H}]^{+}:$311.1067, found: 311.1062 .

$14 H$-Dibenzo[a,c]thioxanthen-14-one (6b). $6 \mathrm{~b}(18 \mathrm{mg}, 58 \%$ yield) was generated following a procedure for the synthesis of 6a as a white solid, mp 198.6-199.4 ${ }^{\circ} \mathrm{C}$. ${ }^{1} \mathrm{H}$ NMR $(400 \mathrm{MHz}$, $\left.\mathrm{CDCl}_{3}\right) \delta 9.59-9.43(\mathrm{~m}, 1 \mathrm{H}), 8.67(\mathrm{~d}, J=8.3 \mathrm{~Hz}, 1 \mathrm{H}), 8.64-8.59$ $(\mathrm{m}, 1 \mathrm{H}), 8.53(\mathrm{~d}, J=7.9 \mathrm{~Hz}, 1 \mathrm{H}), 8.46(\mathrm{~d}, J=8.2 \mathrm{~Hz}, 1 \mathrm{H}), 7.77(\mathrm{t}, J$ $=7.6 \mathrm{~Hz}, 1 \mathrm{H}), 7.73-7.59(\mathrm{~m}, 5 \mathrm{H}), 7.54(\mathrm{t}, J=7.5 \mathrm{~Hz}, 1 \mathrm{H}) \mathrm{ppm}$. ${ }^{13} \mathrm{C}$ NMR $\left(100 \mathrm{MHz}, \mathrm{CDCl}_{3}\right) \delta 182.9,139.2,134.1,132.3,131.7$, $131.6,130.1$, 129.9, 129.5, 129.4, 128.0, 127.7, 127.5, 127.4, 
127.3, 127.2 125.6, 124.9, 124.7, 123.5, 122.6 ppm. HRMS (ESI) $\mathrm{m} / z$ calcd for $\mathrm{C}_{21} \mathrm{H}_{13} \mathrm{OS}[\mathrm{M}+\mathrm{H}]^{+}: 313.0682$, found: 313.0671 .

9-Fluorodibenzo[i,k]phenanthridine (6c). 6c (17 mg, $57 \%$ yield) was generated following a procedure for the synthesis of 6a as a white solid, mp 151.9-152.5 ${ }^{\circ} \mathrm{C} .{ }^{1} \mathrm{H}$ NMR $(400 \mathrm{MHz}$, $\left.\mathrm{CDCl}_{3}\right) \delta 10.04(\mathrm{~s}, 1 \mathrm{H}), 8.88(\mathrm{~d}, J=8.2 \mathrm{~Hz}, 1 \mathrm{H}), 8.82-8.64(\mathrm{~m}$, $3 \mathrm{H}), 8.54(\mathrm{dd}, J=11.2,2.3 \mathrm{~Hz}, 1 \mathrm{H}), 8.31(\mathrm{dd}, J=9.0,6.0 \mathrm{~Hz}, 1 \mathrm{H})$, 7.86-7.69 (m, 4H), 7.61-7.46 (m, 1H) ppm. ${ }^{13} \mathrm{C}$ NMR $(100 \mathrm{MHz}$, $\left.\mathrm{CDCl}_{3}\right) \delta 162.6,160.2,146.2,143.7,132.6,132.0,131.9,130.5$, 129.1, 129.0, 128.5, 128.3, 128.1, 127.6, 127.2, 124.0, 123.5, 123.2, 122.6, 118.4, 118.1, 112.1, $111.8 \mathrm{ppm} .{ }^{19} \mathrm{~F}$ NMR $(376 \mathrm{MHz}$, $\left.\mathrm{CDCl}_{3}\right) \delta-112.6 \mathrm{ppm}$. HRMS (ESI) $m / z$ calcd for $\mathrm{C}_{21} \mathrm{H}_{13} \mathrm{FN}[\mathrm{M}+$ $\mathrm{H}]^{+}:$298.1027, found: 298.1024 .

\section{General procedure for synthesis of 7}

10-(2-(1H-Indol-3-yl)ethyl)chromeno[3,2-b]indol-11(10H)one (7a). 7a (48 mg, 63\% yield) was generated following a procedure for the synthesis of $\mathbf{4 l}$ as a white solid, $\mathrm{mp}$ 157.5158.6 ${ }^{\circ} \mathrm{C} .{ }^{1} \mathrm{H}$ NMR $\left(400 \mathrm{MHz}, \mathrm{CDCl}_{3}\right) \delta 8.55-8.45(\mathrm{~m}, 1 \mathrm{H}), 8.01$ $(\mathrm{d}, J=8.1 \mathrm{~Hz}, 1 \mathrm{H}), 7.97(\mathrm{~s}, 1 \mathrm{H}), 7.81(\mathrm{~d}, J=7.0 \mathrm{~Hz}, 1 \mathrm{H}), 7.76-$ $7.66(\mathrm{~m}, 2 \mathrm{H}), 7.50-7.44(\mathrm{~m}, 1 \mathrm{H}), 7.43-7.38(\mathrm{~m}, 1 \mathrm{H}), 7.35(\mathrm{~d}, J$ $=7.0 \mathrm{~Hz}, 1 \mathrm{H}), 7.29(\mathrm{~d}, J=8.6 \mathrm{~Hz}, 1 \mathrm{H}), 7.25-7.16(\mathrm{~m}, 3 \mathrm{H}), 6.97$ $(\mathrm{d}, J=2.0 \mathrm{~Hz}, 1 \mathrm{H}), 5.09-4.96(\mathrm{~m}, 2 \mathrm{H}), 3.43-3.25(\mathrm{~m}, 2 \mathrm{H}) \mathrm{ppm}$. ${ }^{13} \mathrm{C}$ NMR $\left(100 \mathrm{MHz}, \mathrm{CDCl}_{3}\right) \delta 170.3,155.6,145.2,138.1,136.4$, $132.8,128.1,127.6,126.3,124.4,124.0,122.5,122.2,120.2$, 120.1, 119.7, 118.9, 118.2, 114.9, 112.8, 111.3, 110.4, 100.1, 45.8, $27.1 \mathrm{ppm}$. HRMS (ESI) $\mathrm{m} / z$ calcd for $\mathrm{C}_{25} \mathrm{H}_{19} \mathrm{~N}_{2} \mathrm{O}_{2}[\mathrm{M}+$ $\mathrm{H}]^{+}: 379.1441$, found: 379.1442 .

10-((3R)-10,13-Dimethyl-17-oxohexadecahydro-1H-cyclopenta $[a]$ phenanthren-3-yl)chromeno[3,2-b]indol-11(10H)one (7b). 7 b (57 mg, 56\% yield) was generated following a procedure for the synthesis of 41 as a white solid, mp 167.5-168.6 ${ }^{\circ} \mathrm{C} .{ }^{1} \mathrm{H}$ NMR $\left(400 \mathrm{MHz}, \mathrm{CDCl}_{3}\right) \delta 8.47(\mathrm{~d}, J=$ $7.6 \mathrm{~Hz}, 1 \mathrm{H}), 8.07$ (d, $J=7.5 \mathrm{~Hz}, 1 \mathrm{H}), 7.70(\mathrm{dd}, J=14.6$, $7.1 \mathrm{~Hz}, 2 \mathrm{H}), 7.61(\mathrm{~d}, J=8.2 \mathrm{~Hz}, 1 \mathrm{H}), 7.51(\mathrm{~s}, 1 \mathrm{H}), 7.45(\mathrm{~s}$, $1 \mathrm{H}), 7.27$ (d, $J=9.2 \mathrm{~Hz}, 1 \mathrm{H}), 5.99(\mathrm{~d}, J=6.3 \mathrm{~Hz}, 1 \mathrm{H}), 2.76-$ $2.64(\mathrm{~m}, 1 \mathrm{H}), 2.48(\mathrm{dd}, J=19.2,8.4 \mathrm{~Hz}, 1 \mathrm{H}), 2.27(\mathrm{~s}, 1 \mathrm{H})$, $2.10(\mathrm{dd}, J=19.3,9.6 \mathrm{~Hz}, 1 \mathrm{H}), 2.05-1.91(\mathrm{~m}, 3 \mathrm{H}), 1.84(\mathrm{t}, J=$ $14.4 \mathrm{~Hz}, 2 \mathrm{H}), 1.71(\mathrm{~s}, 5 \mathrm{H}), 1.51(\mathrm{dt}, J=26.0,13.5 \mathrm{~Hz}, 5 \mathrm{H})$, $1.30(\mathrm{~d}, J=8.6 \mathrm{~Hz}, 4 \mathrm{H}), 1.12(\mathrm{~s}, 3 \mathrm{H}), 1.07(\mathrm{~s}, 1 \mathrm{H}), 0.93(\mathrm{~s}$, $3 \mathrm{H}), 0.89$ (d, $J=11.4 \mathrm{~Hz}, 2 \mathrm{H}) \mathrm{ppm} .{ }^{13} \mathrm{C}$ NMR $(100 \mathrm{MHz}$, $\left.\mathrm{CDCl}_{3}\right) \delta 170.2,155.2,145.5,136.9,132.8,127.8,126.4$, 124.5, 123.9, 120.5, 120.3, 120.0, 118.0, 115.7, 112.5, 56.3, 51.6, 50.4, 48.1, 39.6, 36.9, 36.0, 35.6, 34.4, 33.0, 31.9, 30.6, 28.4, 26.3, 21.9, 20.8, 17.4, 14.0 ppm. ${ }^{13} \mathrm{C}$ NMR (100 MHz, dept 90, $\left.\mathrm{CDCl}_{3}\right) \delta 132.7,127.7,126.3,123.8,120.4,120.0$, $117.9,112.4,56.2,51.4,50.3,39.5,35.5 \mathrm{ppm} .{ }^{13} \mathrm{C}$ NMR $(100$ $\mathrm{MHz}$, dept 135, $\left.\mathrm{CDCl}_{3}\right) \delta 132.7,127.7,126.3,123.8,120.4$, $119.9,117.9,112.4,56.2,51.4$, 50.3, 39.5, 36.8, 35.9, 35.5, $32.9,31.8,30.5,28.3,26.2,21.8,20.7,17.3,13.9$ ppm. HRMS (ESI) $m / z$ calcd for $\mathrm{C}_{34} \mathrm{H}_{38} \mathrm{NO}_{3}[\mathrm{M}+\mathrm{H}]^{+}:$508.2846, found: 508.2855 .

10-(2-Aminophenyl)chromeno[3,2-b]indol-11(10H)-one (8a). 8a (43 mg, 66\% yield) was generated following a procedure for the synthesis of 3a as a pink solid, mp 215.5-216.8 ${ }^{\circ} \mathrm{C} .{ }^{1} \mathrm{H}$ NMR $\left(500 \mathrm{MHz}, \mathrm{CDCl}_{3}\right) \delta 8.33(\mathrm{~d}, J=7.2 \mathrm{~Hz}, 1 \mathrm{H}), 8.09(\mathrm{~d}, J=8.0 \mathrm{~Hz}$,
$1 \mathrm{H}), 7.77-7.66(\mathrm{~m}, 2 \mathrm{H}), 7.49(\mathrm{t}, J=7.6 \mathrm{~Hz}, 1 \mathrm{H}), 7.40(\mathrm{dd}, J=14.6$, $6.9 \mathrm{~Hz}, 1 \mathrm{H}), 7.34(\mathrm{dd}, J=9.3,5.4 \mathrm{~Hz}, 2 \mathrm{H}), 7.23(\mathrm{~d}, J=8.5 \mathrm{~Hz}$, $1 \mathrm{H}), 7.19(\mathrm{~d}, J=7.6 \mathrm{~Hz}, 1 \mathrm{H}), 7.01(\mathrm{~d}, J=6.8 \mathrm{~Hz}, 1 \mathrm{H}), 6.91(\mathrm{~s}, 1 \mathrm{H})$, 2.71 (s, 2H). ppm. $\left.{ }^{13} \mathrm{C} \mathrm{NMR} \mathrm{(100} \mathrm{MHz,} \mathrm{CDCl}_{3}\right) \delta 169.3,155.8$, 146.3, 139.0, 133.0, 130.0, 129.6, 128.9, 126.5, 124.6, 124.2, 121.5, 120.1, 119.1, 118.2, 116.9, 115.9, 112.1 ppm. HRMS (ESI) $m / z$ calcd for $\mathrm{C}_{21} \mathrm{H}_{15} \mathrm{~N}_{2} \mathrm{O}_{2}[\mathrm{M}+\mathrm{H}]^{+}$: 327.1128, found: 327.1127 .

10-(2-Aminophenyl)-3-methoxychromeno[3,2-b]indol-11(10H)one (8b). 8 b (48 mg, 68\% yield) was generated following a procedure for the synthesis of $3 \mathrm{a}$ as a white solid, mp $224.2-226.1^{\circ} \mathrm{C} .{ }^{1} \mathrm{H}$ NMR (400 MHz, $\left.\mathrm{CDCl}_{3}\right) \delta 8.38(\mathrm{~d}, J=8.8 \mathrm{~Hz}, 1 \mathrm{H}), 8.20(\mathrm{~d}, J=$ $8.0 \mathrm{~Hz}, 1 \mathrm{H}), 7.62(\mathrm{t}, J=7.6 \mathrm{~Hz}, 1 \mathrm{H}), 7.47(\mathrm{t}, J=7.6 \mathrm{~Hz}, 2 \mathrm{H}), 7.41(\mathrm{~s}$, $1 \mathrm{H}), 7.35$ (dd, $J=12.6,8.3 \mathrm{~Hz}, 2 \mathrm{H}), 7.13(\mathrm{~d}, J=8.9 \mathrm{~Hz}, 1 \mathrm{H}), 7.09$ (d, $J=8.0 \mathrm{~Hz}, 1 \mathrm{H}), 7.04(\mathrm{t}, J=7.5 \mathrm{~Hz}, 1 \mathrm{H}), 4.11(\mathrm{~s}, 3 \mathrm{H}) . \mathrm{ppm} .{ }^{13} \mathrm{C} \mathrm{NMR}$ $\left(100 \mathrm{MHz}, \mathrm{CDCl}_{3}\right) \delta 169.1,163.7,157.5,145.9,144.2,138.6,130.0$, 129.6, 128.5, 127.7, 123.5, 121.3, 121.0, 119.8, 118.9, 118.4, 116.7, 115.9, 113.4, 112.0, 100.6, 56.0 ppm. HRMS (ESI) $\mathrm{m} / \mathrm{z}$ calcd for $\mathrm{C}_{22} \mathrm{H}_{17} \mathrm{~N}_{2} \mathrm{O}_{3}[\mathrm{M}+\mathrm{H}]^{+}:$357.1234, found: 357.1237.

\section{Procedure for the synthesis of $9 a$}

To a stirred solution of $\mathbf{8 a}(40 \mathrm{mg})$ in dichloromethane $(3 \mathrm{~mL})$, was added acetyl chloride (1.2 equiv.) and triethylamine (2.0 equiv.). The reaction proceeded at $\mathrm{rt}$ for $4 \mathrm{~h}$ before dichloromethane was removed by a rotary evaporation. The reaction mixture was extracted with EtOAc. The combined organic layers were washed with $\mathrm{H}_{2} \mathrm{O}$ and brine, dried over anhydrous $\mathrm{Na}_{2} \mathrm{SO}_{4}$, and evaporated in a vacuo. The residue was purified by column chromatography $(\mathrm{PE} / \mathrm{EtOAc}=10 / 1-5 / 1)$ to provide $\mathrm{N}$-(2-(11oxochromeno[3,2- $b]$ indol-10(11H)-yl)phenyl) acetamide (42 mg) as a yellow solid. Then, to this obtained solid was added polyphosphoric acid $(0.2 \mathrm{~mL})$ and $\mathrm{POCl}_{3}$ (10 equiv.). The reaction proceeded in a sealed tube at $120{ }^{\circ} \mathrm{C}$ for $3 \mathrm{~h}$. The reaction mixture was neutralized with $\mathrm{NaHCO}_{3}$ (Sat.) and extracted with EtOAc. The combined organic layers were washed with $\mathrm{H}_{2} \mathrm{O}$ and brine, dried over anhydrous $\mathrm{Na}_{2} \mathrm{SO}_{4}$, and evaporated in a vacuo. The residue was purified by column chromatography (PE/EtOAc $=10 / 1-3 / 1)$ to provide 6-methyl-15H-benzo[2,3][1,4] diazepino $[6,7,1-h, i]$ chromeno $[3,2-b]$ indol-15-one $9 a$ as a yellow solid (33 mg, 51\% yield over two steps), mp 198.2-199.4 ${ }^{\circ} \mathrm{C} .{ }^{1} \mathrm{H}$ NMR $\left(400 \mathrm{MHz}, \mathrm{CDCl}_{3}\right) \delta 8.47(\mathrm{dd}, J=8.0,1.5 \mathrm{~Hz}, 1 \mathrm{H}), 7.93(\mathrm{~d}, J=$ $7.8 \mathrm{~Hz}, 1 \mathrm{H}), 7.81-7.74(\mathrm{~m}, 1 \mathrm{H}), 7.69$ (d, $J=8.4 \mathrm{~Hz}, 1 \mathrm{H}), 7.54$ (d, $J$ $=7.3 \mathrm{~Hz}, 1 \mathrm{H}), 7.53-7.48(\mathrm{~m}, 1 \mathrm{H}), 7.33(\mathrm{t}, J=7.8 \mathrm{~Hz}, 2 \mathrm{H}), 7.17-$ $7.10(\mathrm{~m}, 2 \mathrm{H}), 6.75-6.65(\mathrm{~m}, 1 \mathrm{H}), 2.60$ (s, 3H) ppm. ${ }^{13} \mathrm{C} \mathrm{NMR}(100$ $\left.\mathrm{MHz}, \mathrm{CDCl}_{3}\right) \delta 169.4,167.1,155.5,151.9,151.0,139.7,135.2$, $133.5,129.5$, 129.4, 127.5, 126.9, 125.9, 125.8, 124.9, 124.6, 124.4, 123.8, 122.6, 122.2, 119.8, 118.1, 28.5 ppm. HRMS (ESI) $m / z$ calcd for $\mathrm{C}_{23} \mathrm{H}_{15} \mathrm{~N}_{2} \mathrm{O}_{2}[\mathrm{M}+\mathrm{H}]^{+}$: 351.1128 , found: 351.1127 .

\section{Procedure for the synthesis of $9 b$}

To a stirred solution of $\mathbf{8 b}(45 \mathrm{mg})$ in $\mathrm{EtOH}(4 \mathrm{~mL})$, was added $\mathrm{TsOH} \cdot \mathrm{H}_{2} \mathrm{O}$ (0.1 equiv.). The reaction proceeded at a reflux overnight before EtOH was removed by a rotary evaporation. The reaction mixture was extracted with EtOAc. The combined organic layers were washed with $\mathrm{H}_{2} \mathrm{O}$ and brine, dried over anhydrous $\mathrm{Na}_{2} \mathrm{SO}_{4}$, and evaporated in a vacuo. The residue was purified by column chromatography $(\mathrm{PE} / \mathrm{EtOAc}=10 / 1-5 / 1)$ to 
provide 8-methoxy-10-oxa-5,14b-diazaindeno $\quad[1,2,3-g, h]$ tetraphene 9b as a yellow solid (41 mg, 95\% yield), mp 173.5$174.4{ }^{\circ} \mathrm{C} .{ }^{1} \mathrm{H}$ NMR $\left(400 \mathrm{MHz}, \mathrm{CDCl}_{3}\right) \delta 8.33(\mathrm{~d}, J=8.0 \mathrm{~Hz}, 1 \mathrm{H})$, $8.24(\mathrm{~d}, J=8.6 \mathrm{~Hz}, 1 \mathrm{H}), 8.02(\mathrm{dd}, J=17.2,8.0 \mathrm{~Hz}, 2 \mathrm{H}), 7.75(\mathrm{~d}, J$ $=7.3 \mathrm{~Hz}, 1 \mathrm{H}), 7.58(\mathrm{t}, J=7.8 \mathrm{~Hz}, 1 \mathrm{H}), 7.44(\mathrm{t}, J=7.5 \mathrm{~Hz}, 1 \mathrm{H})$, $7.32(\mathrm{t}, J=7.6 \mathrm{~Hz}, 1 \mathrm{H}), 7.27-7.21(\mathrm{~m}, 1 \mathrm{H}), 6.92(\mathrm{~d}, J=10.1 \mathrm{~Hz}$, $2 \mathrm{H}), 3.93(\mathrm{~s}, 3 \mathrm{H}) \mathrm{ppm} .{ }^{13} \mathrm{C} \mathrm{NMR}\left(100 \mathrm{MHz}, \mathrm{CDCl}_{3}\right) \delta 163.3,158.1$, $148.0,139.5,132.8,131.2,130.3,129.1,126.3,125.8,125.1$, $124.6,121.6,119.2$, 118.1, 116.7, 114.8, 114.0, 113.6, 112.4, 102.2, $55.8 \mathrm{ppm}$. HRMS (ESI) $m / z$ calcd for $\mathrm{C}_{22} \mathrm{H}_{15} \mathrm{~N}_{2} \mathrm{O}_{2}[\mathrm{M}+\mathrm{H}]^{+}$: 339.1128, found: 339.1122 .

\section{The general synthesis of heterocyclic iodoniums 1}

All the synthetic heterocyclic idoniums are reported in our previous work, and they are prepared conveniently using reported procedure. ${ }^{10,18}$

11-Oxo-11H-benzo[b]chromeno[2,3-d]iodol-10-ium triflate (1a). ${ }^{1} \mathrm{H}$ NMR (400 MHz, DMSO) $\delta 8.50-8.35$ (m, 2H), 8.22 (dd, $J$ $=7.9,1.4 \mathrm{~Hz}, 1 \mathrm{H}), 8.11-8.02(\mathrm{~m}, 2 \mathrm{H}), 8.01-7.91(\mathrm{~m}, 2 \mathrm{H}), 7.72(\mathrm{t}, J$ $=7.5 \mathrm{~Hz}, 1 \mathrm{H}) \mathrm{ppm} .{ }^{13} \mathrm{C} \mathrm{NMR}(100 \mathrm{MHz}, \mathrm{DMSO}) \delta 172.3,164.1$, $155.2,135.9$, 135.2, 134.7, 131.4, 131.3, 128.9, 127.0, 125.2, 122.5, 119.6, 118.8, 108.4 ppm.

8-Methyl-11-oxo-10 $\lambda^{3}$-benzo $[b]$ chromeno[2,3- $\left.d\right]$ iodol-10(11H)-yl trifluoromethanesulfonate (1b). ${ }^{1} \mathrm{H}$ NMR (400 MHz, DMSO) $\delta 8.31$ $(\mathrm{d}, J=8.0 \mathrm{~Hz}, 1 \mathrm{H}), 8.23(\mathrm{~s}, 1 \mathrm{H}), 8.21(\mathrm{dd}, J=8.0,1.5 \mathrm{~Hz}, 1 \mathrm{H}), 8.03$ (ddd, $J=8.6,7.1,1.7 \mathrm{~Hz}, 1 \mathrm{H}), 7.95(\mathrm{~d}, J=7.9 \mathrm{~Hz}, 1 \mathrm{H}), 7.88$ (d, $J=$ $7.7 \mathrm{~Hz}, 1 \mathrm{H}), 7.74-7.68$ (m, 1H), 2.58 (s, 3H) ppm. ${ }^{13} \mathrm{C}$ NMR (100 MHz, DMSO) $\delta 172.2,164.0,155.1,146.2,135.8,132.5,132.2,131.2$, $128.5,126.9,125.2,122.4,119.7,118.7,107.4,21.8 \mathrm{ppm}$.

8-Chloro-11-oxo-10 $\lambda^{3}$-benzo $[b]$ chromeno $[2,3-d]$ iodol-10(11H)-yl trifluoromethanesulfonate (1c). ${ }^{1} \mathrm{H}$ NMR (400 MHz, DMSO) $\delta 8.42$ $(\mathrm{d}, J=1.8 \mathrm{~Hz}, 1 \mathrm{H}), 8.39(\mathrm{~d}, J=8.4 \mathrm{~Hz}, 1 \mathrm{H}), 8.21(\mathrm{dd}, J=7.9,1.2 \mathrm{~Hz}$, $1 \mathrm{H}), 8.14$ (dd, $J=8.4,1.8 \mathrm{~Hz}, 1 \mathrm{H}), 8.07-8.00$ (m, 1H), 7.95 (d, $J=$ $8.3 \mathrm{~Hz}, 1 \mathrm{H}), 7.71(\mathrm{t}, J=7.5 \mathrm{~Hz}, 1 \mathrm{H}) \mathrm{ppm} .{ }^{13} \mathrm{C}$ NMR $(100 \mathrm{MHz}$, DMSO) $\delta$ 172.2, 163.2, 155.1, 138.5, 136.0, 134.3, 131.9, 131.3, 130.9, 129.8, 128.5, 127.1, 125.3, 122.5, 120.1, 118.8, 109.1 ppm.

8-Fluoro-11-oxo-10 $\lambda^{3}$-benzo $[b]$ chromeno $[2,3-d]$ iodol-10(11H)-yl trifluoromethanesulfonate (1d). ${ }^{1} \mathrm{H}$ NMR (400 MHz, DMSO) $\delta 8.50-8.44(\mathrm{~m}, 1 \mathrm{H}), 8.22$ (ddd, $J=8.0,5.9,2.3 \mathrm{~Hz}, 2 \mathrm{H}), 8.09-8.00$ $(\mathrm{m}, 1 \mathrm{H}), 8.01-7.91(\mathrm{~m}, 2 \mathrm{H}), 7.71(\mathrm{dd}, J=10.6,4.3 \mathrm{~Hz}, 1 \mathrm{H}) \mathrm{ppm} .{ }^{13} \mathrm{C}$ NMR (100 MHz, DMSO) $\delta$ 172.1, 165.2, 163.2, 162.7, 155.2, 136.0, 132.1, 130.8, 130.7, 127.1, 125.3, 122.5, 120.3, 120.2, 112.0, 119.7, 119.3, 119.0, 118.8, $108.5 \mathrm{ppm}$.

7-Fluoro-11-oxo-10 $\lambda^{3}$-benzo $[b]$ chromeno[2,3- $\left.d\right]$ iodol-10(11H)-yl trifluoromethanesulfonate (1e). ${ }^{1} \mathrm{H}$ NMR (400 MHz, DMSO) $\delta 8.50-$ $8.43(\mathrm{~m}, 1 \mathrm{H}), 8.39$ (dd, $J=5.6,2.7 \mathrm{~Hz}, 1 \mathrm{H}), 8.23(\mathrm{~d}, J=7.9 \mathrm{~Hz}, 1 \mathrm{H})$, $8.06(\mathrm{t}, J=7.5 \mathrm{~Hz}, 1 \mathrm{H}), 7.95(\mathrm{dd}, J=8.2,2.3 \mathrm{~Hz}, 1 \mathrm{H}), 7.87$ (ddd, $J=$ 8.9, 6.2, $2.7 \mathrm{~Hz}, 1 \mathrm{H}), 7.73(\mathrm{t}, J=6.2 \mathrm{~Hz}, 1 \mathrm{H}) \mathrm{ppm} .{ }^{13} \mathrm{C} \mathrm{NMR}(100$ MHz, DMSO) $\delta 172.3,164.9,163.0,162.4,155.1,137.5,137.4,136.1$, 133.7, 133.6, 127.1, 125.3, 122.4, 122.3, 122.0, 118.8, 115.9, 115.7, 113.6, $109.7 \mathrm{ppm}$.

11-Oxo-10 $\lambda^{3}$-benzo[ $[b]$ thiochromeno[2,3- $\left.d\right]$ iodol-10 $(11 H)$ yl trifluoromethanesulfonate (1f). ${ }^{1} \mathrm{H}$ NMR (400 MHz, DMSO) $\delta 8.46(\mathrm{t}, J=9.1 \mathrm{~Hz}, 2 \mathrm{H}), 8.34(\mathrm{~d}, J=7.8 \mathrm{~Hz}, 1 \mathrm{H}), 8.22(\mathrm{~d}, J=$ $8.1 \mathrm{~Hz}, 1 \mathrm{H}), 8.04(\mathrm{~d}, J=7.6 \mathrm{~Hz}, 1 \mathrm{H}), 8.01-7.96(\mathrm{~m}, 1 \mathrm{H}), 7.92(\mathrm{t}$, $J=7.9 \mathrm{~Hz}, 1 \mathrm{H}), 7.87(\mathrm{t}, J=7.6 \mathrm{~Hz}, 1 \mathrm{H}) \mathrm{ppm} .{ }^{13} \mathrm{C} \mathrm{NMR}(100$
MHz, DMSO) $\delta 174.9,150.6,143.4,135.8,134.0,132.1,131.5$, $129.7,128.9,128.8,128.1,128.0,123.3,122.8$ ppm.

$7 \boldsymbol{H}-7 \boldsymbol{\lambda}^{3}$-Benzo[4,5]iodolo[2,3-c]quinolin-7-yl trifluoromethanesulfonate (1g). To a stirred solution of 3-iodo-4phenylquinoline 1g-l (2.0 g, $6.04 \mathrm{~mol})$ in anhydrous DCM (20 $\mathrm{mL}$ ) was added $\mathrm{TfOH}(1.60 \mathrm{~mL}, 3.0$ equiv.) and followed by the slow addition of $m$-CPBA $(85 \%, 1.84 \mathrm{~g}, 1.5$ equiv.). The solution was stirred for $2 \mathrm{~h}$ at rt before DCM was removed by rotary evaporation. $\mathrm{Et}_{2} \mathrm{O}(20 \mathrm{~mL})$ was added to the remained solid. The mixture was stirred for $30 \mathrm{~min}$, and then filtered. The obtained solid was washed with $\mathrm{Et}_{2} \mathrm{O}$ three times and dried in high vacuo to provide $1 \mathrm{~g}$ ( $2.43 \mathrm{~g}, 84 \%$ yield) as a yellow solid. ${ }^{1} \mathrm{H}$ NMR (400 MHz, DMSO) $\delta 9.53(\mathrm{~s}, 1 \mathrm{H}), 9.14(\mathrm{~d}, J=7.9 \mathrm{~Hz}, 1 \mathrm{H}), 9.09$ (d, $J=$ $8.8 \mathrm{~Hz}, 1 \mathrm{H}), 8.45$ (d, $J=7.6 \mathrm{~Hz}, 1 \mathrm{H}), 8.30$ (d, $J=7.9 \mathrm{~Hz}, 1 \mathrm{H}), 8.03$ $(\mathrm{t}, J=7.6 \mathrm{~Hz}, 2 \mathrm{H}), 7.97-7.86(\mathrm{~m}, 2 \mathrm{H}) \mathrm{ppm} .{ }^{13} \mathrm{C}$ NMR $(100 \mathrm{MHz}$, DMSO) $\delta$ 148.6, 147.9, 144.8, 141.2, 132.4, 132.3, 131.2, 131.1, 131.1, 131.0, 130.6, 129.1, 126.4, 124.2, 123.9, 117.7 ppm.

9-Methyl-7H-7 $\lambda^{3}$-benzo[4,5]iodolo[2,3-c]quinolin-7-yl trifluoromethanesulfonate (1h). ${ }^{1} \mathrm{H}$ NMR (400 MHz, DMSO) $\delta 9.48$ $(\mathrm{s}, 1 \mathrm{H}), 9.04(\mathrm{~d}, J=8.4 \mathrm{~Hz}, 1 \mathrm{H}), 8.99$ (d, $J=8.4 \mathrm{~Hz}, 1 \mathrm{H}), 8.28$ (dd, $J=8.4,1.0 \mathrm{~Hz}, 1 \mathrm{H}), 8.20(\mathrm{~s}, 1 \mathrm{H}), 8.02(\mathrm{dd}, J=11.3,4.0 \mathrm{~Hz}, 1 \mathrm{H})$, $7.95-7.88(\mathrm{~m}, 1 \mathrm{H}), 7.81$ (d, $J=7.5 \mathrm{~Hz}, 1 \mathrm{H}), 2.56$ (s, 3H) ppm. ${ }^{13} \mathrm{C}$ NMR (100 MHz, DMSO) $\delta$ 148.4, 147.7, 144.8, 143.2, 138.4, $132.0,131.9,131.1,131.0,130.5,129.0,126.2$, 124.2, 124.0, 122.4, 119.1, 117.0, $21.2 \mathrm{ppm}$.

9-Chloro-7H-7 $\lambda^{3}$-benzo[4,5]iodolo[2,3-c]quinolin-7-yl trifluoromethanesulfonate (1i). ${ }^{1} \mathrm{H}$ NMR (400 MHz, DMSO) $\delta 9.46$ (s, 1H), 9.03 (d, $J=8.9 \mathrm{~Hz}, 1 \mathrm{H}), 8.94$ (d, $J=8.5 \mathrm{~Hz}, 1 \mathrm{H}), 8.38$ (d, $J$ $=2.2 \mathrm{~Hz}, 1 \mathrm{H}), 8.27(\mathrm{dd}, J=8.4,1.1 \mathrm{~Hz}, 1 \mathrm{H}), 8.05-7.97(\mathrm{~m}, 2 \mathrm{H})$, 7.90 (ddd, $J=8.4,7.0,1.3 \mathrm{~Hz}, 1 \mathrm{H}) \mathrm{ppm} .{ }^{13} \mathrm{C} \mathrm{NMR}(100 \mathrm{MHz}$, DMSO) $\delta$ 148.4, 147.9, 143.8, 140.1, 136.2, 133.2, 131.4, 130.7, 130.5, 129.3, 126.2, 124.9, 124.1, 122.4, 119.1, $118.2 \mathrm{ppm}$.

2-Fluoro-7H-7 $\lambda^{3}$-benzo[4,5]iodolo[2,3-c]quinolin-7-yl trifluoromethanesulfonate (1j). ${ }^{1} \mathrm{H}$ NMR (400 MHz, DMSO) $\delta 9.54$ $(\mathrm{d}, J=3.4 \mathrm{~Hz}, 1 \mathrm{H}), 9.15(\mathrm{dd}, J=7.7,2.7 \mathrm{~Hz}, 1 \mathrm{H}), 8.89-8.72(\mathrm{~m}$, $1 \mathrm{H}), 8.46$ (dd, $J=8.1,2.3 \mathrm{~Hz}, 1 \mathrm{H}), 8.40$ (ddd, $J=9.3,6.0,3.4 \mathrm{~Hz}$, $1 \mathrm{H}), 8.08-7.85(\mathrm{~m}, 3 \mathrm{H}) \mathrm{ppm} .{ }^{13} \mathrm{C}$ NMR (100 MHz, DMSO) $\delta 162.4,159.9,148.0,145.3,144.5,144.4,140.7,133.7,133.6$, $132.4,132.2$, 131.2, 131.2, 127.0, 126.9, 123.7, 122.3, 121.2, 121.0, 119.1, 118.9, 108.8, 108.6 ppm.

Benzo[4,5]iodolo[3,2-c]isoquinolin-11-ium triflate $(1 \mathrm{k}) \cdot{ }^{1} \mathrm{H}$ NMR (400 MHz, DMSO) $\delta 9.73(\mathrm{~s}, 1 \mathrm{H}), 8.59-8.51(\mathrm{~m}, 2 \mathrm{H}), 8.43$ (dd, $J=10.6,8.3 \mathrm{~Hz}, 2 \mathrm{H}), 8.18-8.07(\mathrm{~m}, 1 \mathrm{H}), 7.97(\mathrm{t}, J=7.5 \mathrm{~Hz}$, $2 \mathrm{H}), 7.90-7.81(\mathrm{~m}, 1 \mathrm{H}) \mathrm{ppm} .{ }^{13} \mathrm{C}$ NMR (100 MHz, DMSO) $\delta$ 155.6, 153.4, 140.6, 134.5, 133.6, 132.6, 131.1, 130.7, 130.1, 129.5, 129.3, 126.7, 122.3, 121.4, 120.5 ppm.

9-Methyl-11H-11 $\lambda^{3}$-benzo[4,5]iodolo[3,2-c]isoquinolin-11-yl trifluoromethanesulfonate (11). ${ }^{1} \mathrm{H}$ NMR (400 MHz, DMSO) $\delta 9.58(\mathrm{~s}, 1 \mathrm{H}), 8.40(\mathrm{~d}, J=8.2 \mathrm{~Hz}, 1 \mathrm{H}), 8.34(\mathrm{~d}, J=8.1 \mathrm{~Hz}, 1 \mathrm{H})$, $8.24(\mathrm{~d}, J=7.9 \mathrm{~Hz}, 1 \mathrm{H}), 8.07(\mathrm{~s}, 1 \mathrm{H}), 8.06-8.01(\mathrm{~m}, 1 \mathrm{H}), 7.89(\mathrm{t}, J$ $=7.3 \mathrm{~Hz}, 1 \mathrm{H}), 7.66(\mathrm{~d}, J=7.8 \mathrm{~Hz}, 1 \mathrm{H}), 2.51(\mathrm{~s}, 3 \mathrm{H}) \mathrm{ppm} .{ }^{13} \mathrm{C}$ NMR (100 MHz, DMSO) $\delta$ 155.4, 153.2, 143.2, 137.8, 134.3, 133.5 , 132.0, 130.2, 129.8, 129.4, 129.2, 128.6, 126.4, 121.2, $119.5,21.5 \mathrm{ppm}$.

6-Oxo- $7 \lambda^{3}$-benzo $[b]$ chromeno $[4,3-d]$ iodol-7 $(6 H)$-yl trifluoromethanesulfonate (1m). ${ }^{1} \mathrm{H}$ NMR (400 $\left.\mathrm{MHz}, \mathrm{DMSO}\right)$ $\delta$ 9.12-9.02 (m, 1H), 8.81 (d, $J=7.5 \mathrm{~Hz}, 1 \mathrm{H}), 8.53$ (dd, $J=8.3$, 
$1.0 \mathrm{~Hz}, 1 \mathrm{H}), 8.10-8.01(\mathrm{~m}, 1 \mathrm{H}), 8.00-7.93(\mathrm{~m}, 1 \mathrm{H}), 7.92-7.85(\mathrm{~m}$, $1 \mathrm{H}), 7.71(\mathrm{dd}, J=8.3,0.9 \mathrm{~Hz}, 1 \mathrm{H}), 7.66-7.56(\mathrm{~m}, 1 \mathrm{H}) \mathrm{ppm} .{ }^{13} \mathrm{C}$ NMR (100 MHz, DMSO) $\delta$ 157.2, 153.6, 152.7, 141.0, 133.5, 133.4 , 133.0, 132.2, 131.4, 126.2, 125.6, 125.2, 118.2, 117.9, $117.3 \mathrm{ppm}$.

\section{Conflicts of interest}

There are no conflicts to declare.

\section{Acknowledgements}

We are grateful to the grant support from National Natural Science Foundation of China (81672952, 81872440), Guangdong Science and Technology Program (2017A020215198, 2018A030310240), and Guangzhou Science and Technology Program (201807010041).

\section{Notes and references}

1 (a) H. Ito, K. Ozaki and K. Itami, Angew. Chem. Int. Ed., 2017, 56, 11144-11164; Angew. Chem., 2017, 129, 11296-11317; (b) J. Mei, N. L. C. Leung, R. T. K. Kwok, J. W. Y. Lam and B. Z. Tang, Chem. Rev., 2015, 115, 11718-11940.

2 D. A. Erlanson, S. W. Fesik, R. E. Hubbard, W. Jahnke and H. Jhoti, Nat. Rev. Drug Discovery, 2016, 15, 605-619.

3 (a) G. B. Deng, Z. Q. Wang, J. D. Xia, P. C. Qian, R. J. Song, M. Hu, L. B. Gong and J. H. Li, Angew. Chem. Int. Ed., 2013, 52, 1535-1538; Angew. Chem., 2013, 125, 1575-1578; (b) J. H. Kim, J. Bouffard and S. G. Lee, Angew. Chem. Int. Ed., 2014, 53, 6435-6438; Angew. Chem., 2014, 126, 6553-6556; (c) J. H. Kim, Y. O. Ko, J. Bouffard and S. G. Lee, Chem. Soc. Rev., 2015, 44, 2489-2507; (d) Y. Liu and J.-P. Wan, Org. Biomol. Chem., 2011, 9, 6873-6894.

4 M. Wang, Q. Fan and X. Jiang, Org. Lett., 2018, 20, 216-219. 5 (a) Y. Wu, X. Peng, B. Luo, F. Wu, B. Liu, F. Song, P. Huang and S. Wen, Org. Biomol. Chem., 2014, 12, 9777-9780; (b) D. Zhu, Q. Liu, B. Luo, M. Chen, R. Pi, P. Huang and S. Wen, Adv. Synth. Catal., 2013, 355, 2172-2178.

6 (a) X. Peng, H. Luo, F. Wu, D. Zhu, A. Ganesan, P. Huang and S. Wen, Adv. Synth. Catal., 2017, 359, 1152-1156; (b) D. Zhu, Y. Wu, B. Wu, B. Luo, A. Ganesan, F. H. Wu, R. Pi, P. Huang and S. Wen, Org. Lett., 2014, 16, 2350-2353.

7 Z. Liu, D. Zhu, B. Luo, N. Zhang, Q. Liu, Y. Hu, R. Pi, P. Huang and S. Wen, Org. Lett., 2014, 16, 5600-5603.

8 (a) D. Zhu, M. Li, Z. Wu, Y. Du, B. Luo, P. Huang and S. Wen, Eur. J. Org. Chem., 2019, 4566-4571; (b) B. Luo, Q. Cui, H. Luo, Y. Hu, P. Huang and S. Wen, Adv. Synth. Catal, 2016, 358, 2733-2738; (c) Q. Tan, D. Zhou, T. Zhang, B. Liu and B. Xu, Chem. Commun., 2017, 53, 10279-10282; (d) M. Wang, J. Wei, Q. Fan and X. Jiang, Chem. Commun.,
2017, 53, 2918-2921; (e) P. S. Postnikov, O. A. Guselnikova, M. S. Yusubov, A. Yoshimura, V. N. Nemykin and V. V. Zhdankin, J. Org. Chem., 2015, 80, 5783-5788; (f) Y. A. Vlasenko, P. S. Postnikov, M. E. Trusova, A. Shafir, V. V. Zhdankin, A. Yoshimura and M. S. Yusubov, J. Org. Chem., 2018, 83, 12056-12070.

9 J. Letessier and H. Detert, Synthesis, 2012, 44, 290-296.

10 D. Zhu, Z. Wu, B. Luo, Y. Du, P. Liu, Y. Chen, Y. Hu, P. Huang and S. Wen, Org. Lett., 2018, 20, 4815-4818.

11 (a) S. U. Dighe, S. Khan, I. Soni, P. Jain, S. Shukla, R. Yadav, P. Sen, S. M. Meeran and S. Batra, J. Med. Chem., 2015, 58, 3485-3499; (b) T. K. Mazu, J. R. Etukala, M. R. Jacob, S. I. Khan, L. A. Walker and S. Y. Ablordeppey, Eur. J. Med. Chem., 2011, 46, 2378-2385; (c) G. Van Baelen, S. Hostyn, L. Dhooghe, P. Tapolcsányi, P. Mátyus, G. Lemière, R. Dommisse, M. Kaiser, R. Brun, P. Cos, L. Maes, G. Hajós, Z. Riedl, I. Nagy, B. U. W. Maes and L. Pieters, Bioorg. Med. Chem., 2009, 17, 7209-7217.

12 (a) J. Reis, A. Gaspar, N. Milhazes and F. Borges, J. Med. Chem., 2017, 60, 7941-7957; (b) R. S. Keri, S. Budagumpi, R. K. Pai and R. G. Balakrishna, Eur. J. Med. Chem., 2014, 78, 340-374.

13 (a) Shaveta, S. Mishra and P. Singh, Eur. J. Med. Chem., 2016, 124, 500-536; (b) C. de Bodinat, B. Guardiola-Lemaitre, E. Mocaer, P. Renard, C. Munoz and M. J. Millan, Nat. Rev. Drug Discovery, 2010, 9, 628-642; (c) F. H. Havaldar, S. Bhise and S. Burudkar, J. Serb. Chem. Soc., 2004, 69, 527-532; (d) L. Duan, J. Qiao, Y. Sun and Y. Qiu, $A d v$. Mater., 2011, 23, 1137-1144.

14 (a) Y. Wei, P. Hu, M. Zhang and W. Su, Chem. Rev., 2017, 117, 8864-8907; (b) N. Rodriguez and L. Goossen, Chem. Soc. Rev., 2011, 40, 5030-5048.

15 (a) S. Yang, F. Wang, Y. Wu, W. Hua and F. Zhang, Org. Lett., 2018, 20, 1491-1495; (b) S. Yang, W. Hua, Y. Wu, T. Hu, F. Wang, X. Zhang and F. Zhang, Chem. Commun., 2018, 54, 3239-3242.

16 (a) S. Sergeyev, W. Pisulab and Y. Geerts, Chem. Soc. Rev., 2007, 36, 1902-1929; (b) S. Laschat, A. Baro, N. Steinke, F. Giesselmann, C. Hgele, G. Scalia, R. Judele, E. Kapatsina, S. Sauer, A. Schreivogel and M. Tosoni, Angew. Chem. Int. Ed., 2007, 46, 4832-4887; Angew. Chem., 2007, 119, 4916-4973.

17 J. Wei, B. Han, Q. Guo, X. Shi, W. Wang and N. Wei, Angew. Chem. Int. Ed., 2010, 49, 8209-8213; Angew. Chem., 2010, 122, 8385-8389.

18 (a) C. Zhou, A. Dubrovsky and R. Larock, J. Org. Chem., 2006, 71, 1626-1632; (b) Q. Huang, J. Hunter and R. Larock, J. Org. Chem., 2002, 67, 3437-3444; (c) M. Reddy, N. Thirupathi, M. Babu and S. Puri, J. Org. Chem., 2013, 78, 5878-5888. 